This is a preprint version. The original publication is available at www.springerlink.com

\title{
PERIODIC PLUS SMOOTH IMAGE DECOMPOSITION
}

\author{
Lionel Moisan \\ Université Paris Descartes \\ MAP5, CNRS UMR 8145 \\ Lionel.Moisan@parisdescartes.fr
}

\begin{abstract}
When the Discrete Fourier Transform of an image is computed, the image is implicitly assumed to be periodic. Since there is no reason for opposite borders to be alike, the "periodic" image generally presents strong discontinuities across the frame border. These edge effects cause several artifacts in the Fourier Transform, in particular a well-known "cross" structure made of high energy coefficients along the axes, which can have strong consequences on image processing or image analysis techniques based on the image spectrum (including interpolation, texture analysis, image quality assessment, etc.). In this paper, we show that an image can be decomposed into a sum of a "periodic component" and a "smooth component", which brings a simple and computationally efficient answer to this problem. We discuss the interest of such a decomposition on several applications.
\end{abstract}

keywords: Discrete Fourier Transform, periodic image, edge effect, ringing, artifact, sinc interpolation, image quality assessment, phase coherence.

\section{Introduction}

The Fourier Transform plays a key role in several major image processing issues. First, ShannonWhittaker Sampling Theorem [23] gives a nice equivalence between continuous band-limited images and discrete images, which has consequences on image sampling and the aliasing phenomenon, image interpolation, geometrical transforms, etc. Second, since any continuous linear translation-invariant operator on finite-energy $\left(L^{2}\right)$ functions boils down to a multiplication in Fourier domain, many filtering issues are better understood and processed in Fourier domain, leading to classical applications in image smoothing, image deblurring, and pattern recognition [25] for example. Third, the Fourier Transform, due to its global nature, can be used in various issues like image registration $[8,15,16,17,21,26]$, image quality assessment $[2,4,5,19,20]$, watermarking [12, 18], document analysis [14], non-linear interpolation [10, 29], etc.

In practice, since real data are made of discrete finite images, the Discrete Fourier Transform (DFT) is used. A good point is that the DFT can be computed efficiently for images whose dimensions can be written as products of small prime integers, reducing the theoretical $O\left(N^{3}\right)$ cost (using separability) to $O\left(N^{2} \cdot \log N\right)$ (using the Fast Fourier Transform) for a $N \times N$ image when $N$ is a power of two. A bad point is that the DFT is nothing but the continuous Fourier 
Transform of a periodized image obtained by tiling the whole plane with side-to-side copies of the original discrete image. Since there is no reason in general for the opposite borders of an image to be alike, such a periodization tends to produce discontinuities in the image domain, which causes an artificial "cross structure" (as noticed in $[12,14,17,18]$ for example), that is, high-energy coefficients along the axes in Fourier domain that can be responsible for undesirable artifacts in applications.

Several solutions have been proposed to overcome these edge effects. The most classical way consists in forcing smooth transitions at the image borders by multiplying the initial image with an attenuation function that vanishes at the frame border. Many such windowing functions have been proposed to do this: Bartlett, Blackman, Hamming, Hanning, Kaiser, etc. [11]. These approaches can be used to estimate global properties of the Fourier spectrum of an image, provided that the image values near the frame borders can be neglected. However, they are not relevant for most image operations involving the Fourier spectrum (e.g., interpolation) not to mention the skills required to choose the most appropriate windowing function (for given applications or data).

Another popular way to avoid edge effects is to symmetrize the $N \times N$ image into a $2 N \times 2 N$ image, on which the DFT is then applied. Since the opposite borders of the symmetrized image are the same, no edge effects occur in the periodization process (to be more exact, discontinuities are relocated to the first derivative, which causes the "cross structure" to disappear thanks to a much faster decay of Fourier coefficients along the axes). To avoid the multiplication of data size by 4 , it is interesting to notice that this prior symmetrization process amounts to replace the DFT of the image by its Discrete Cosine Transform, even if the latter is sometimes less convenient to use. The most important drawback of the prior symmetrization is that it does much more to the image spectrum than removing the cross structure artifact: it makes the DFT symmetric and real-valued, which can be dramatic if the phase component of the Fourier Transform has to be analyzed, or if orientation issues are concerned.

Several years ago, Beaudoin and Beauchemin [3] proposed to replace the DFT of an image by a better estimate of its continuous Fourier Transform, using regularity assumptions on the image itself. Unfortunately, their method is not broadly applicable because natural images are not very smooth in general (they tend to present discontinuities due to scene occlusions). Moreover, their algorithm requires the estimation of high-order derivatives on the image frame border, which can be an important issue, especially in the presence of noise. Notice that the point of view we take here is more to avoid the DFT artifacts than to define a better discrete estimate of the "real" Fourier Transform. More recently, Saito and Remy [22] and then Zhao, Saito and Wang [28] proposed to avoid edge effects by subtracting a residue to the original image. The "clean" image is obtained by solving a generalized Laplace Equation with prescribed boundary values, using an accurate and fast $\left(O\left(N^{2} \log N\right)\right.$ for a $N \times N$ image $\left.)\right)$ solver derived from [1]. This operation, called Polyharmonic Local Sine Transform (PHLST, and PHLST5 in the second paper), was primarily intended for local Fourier analysis (obtained by cutting the image in small adjacent patches), but could be applied on the entire image as well. However, the imposed constraints (the approximate image must vanish at the frame border) make this method not very well suited for the artifact removal issue we consider here.

In this paper, we propose to decompose an image globally into the sum of a "periodic" component (that is, an image resembling the original image but whose DFT does not suffer from edge effects) and a smooth component that has very slow variations inside the image 
domain. The plan of this paper is the following. In Section 2, we set some notations concerning the DFT and discuss the edge effects it produces, as well as the effect of symmetrization. In Section 3, we introduce the "periodic plus smooth decomposition" in a variational setting and derive three direct formulations: in the image domain, in the Fourier domain, and in both domains. We also give an interpretation in terms of Poisson's equation, and derive a simple and exact algorithm involving a single DFT to compute the decomposition in Fourier Domain. We also establish several properties of the "periodic component" operator. Some applications of the periodic plus smooth decomposition are then described in Section 4 and illustrated with numerical experiments, before we conclude in Section 5 .

\section{Discrete Fourier Transform and edge effects}

Let us consider a discrete gray-level image, that is a function $u: \Omega \rightarrow \mathbb{R}$ defined on a finite domain $\Omega=\{0, \ldots, M-1\} \times\{0, \ldots, N-1\}$. The DFT of $u$ is the discrete function $\hat{u}: \mathbb{Z}^{2} \rightarrow \mathbb{C}$ defined by

$$
\forall(q, r) \in \mathbb{Z}^{2}, \quad \hat{u}(q, r)=\sum_{(k, l) \in \Omega} u(k, l) \exp \left[-2 i \pi\left(\frac{q k}{M}+\frac{r l}{N}\right)\right] .
$$

The function $\hat{u}$ is $(M, N)$-periodic, that is, $M$-periodic with respect to its first argument and $N$ periodic with respect to its second argument. Since $u$ is real-valued, $\hat{u}$ is conjugate-symmetric, that is

$$
\forall(q, r) \in \mathbb{Z}^{2}, \quad \hat{u}(-q,-r)=u^{*}(q, r),
$$

where $z^{*}$ denotes the conjugate complex of $z \in \mathbb{C}$. From these properties, it can be easily deduced that the complete description of $\hat{u}$ only involves $M N$ real numbers.

Using Cooley and Tukey's fast algorithm [7], the famous Fast Fourier Transform (FFT), the numerical computation of $\hat{u}$ can be done in $2 M N(\phi(M)+\phi(N))$ operations (additions and multiplications), where the function $\phi$ is defined by

$$
\phi\left(\prod_{i \in I} p_{i}\right)=\sum_{i \in I}\left(p_{i}-1\right)
$$

for any finite sequence of prime numbers $\left(p_{i}\right)_{i \in I}$. This algorithm is particularly efficient when $M$ and $N$ are powers of two (in this case, the computational cost is $2 M N\left(\log _{2} M+\log _{2} N\right)$ ), but it remains very efficient for more general image dimensions, provided that the prime factor decomposition of $M$ and $N$ involve many small factors. This hypothesis is not always satisfied ( $M$ and $N$ could be prime numbers for example), but it can be easily forced as soon as a loss of a few lines and columns (image cropping) is acceptable.

\subsection{Implicit periodization}

The DFT can be interpreted as the continuous Fourier Transform of a periodic distribution. Let $u: \Omega \rightarrow \mathbb{R}$ be a discrete $M \times N$ image, extended to $\dot{u}: \mathbb{Z}^{2} \rightarrow \mathbb{R}$ by $(M, N)$ periodicity. Consider the associated periodic distribution

$$
U=\sum_{(k, l) \in \mathbb{Z}^{2}} \dot{u}(k, l) \delta_{(k, l)},
$$


where $\delta_{\mathbf{x}}$ is the Dirac distribution at point $\mathbf{x} \in \mathbb{R}^{2}$. Then, the continuous Fourier Transform of $U$ (in the distributional sense) is simply

$$
\widehat{U}=\frac{4 \pi^{2}}{M N} \sum_{(q, r) \in \mathbb{Z}^{2}} \hat{u}(q, r) \delta_{(2 \pi q / M, 2 \pi r / N)}
$$

Hence, the continuous Fourier Transform of the periodized image $U$ is supported by a discrete grid (equivalent to $\mathbb{Z}^{2}$, after rescaling), and its coefficients are given by the DFT of $u$. Another way to understand the implicit periodization in (1) is to notice that for any discrete rectangle $\Omega^{\prime} \subset \mathbb{Z}^{2}$ obtained by translating $\Omega$, we have

$$
\forall(q, r) \in \mathbb{Z}^{2}, \quad \hat{u}(q, r)=\sum_{(k, l) \in \Omega^{\prime}} \dot{u}(k, l) \exp \left[-2 i \pi\left(\frac{q k}{M}+\frac{r l}{N}\right)\right] .
$$

Considering discrete images as periodic (which they are not in general) produces gray-level gaps (roughly speaking, discrete "discontinuities") at the boundary of the frame border, since there is no reason in general for opposite pixels of an image to have similar gray values, as illustrated on Figure 1 (top right image). When computing the Fourier Transform of $U$, the horizontal and vertical gaps resulting from the image periodization $(u \rightarrow \dot{u})$ are responsible for phase-coherent high-frequency components along the axes in the frequency domain. Since $\widehat{U}$ and $\hat{u}$ are essentially the same function (as shown in Equation 2), this artifact also happens for $\hat{u}$, and is noticeable as an artificial "cross structure" both on the modulus component (Figure 1, bottom left) and on the phase component (Figure 1, bottom right).

As we shall see later in Section 4, not only the implicit periodization realized by the DFT introduces visual bias in the Fourier Transform, but it also has undesirable consequences for several applications, including interpolation (appearance of ringing artifacts), texture analysis (wrong Fourier coefficients along the axes), estimation of image smoothness (linked to the decay rate of the DFT modulus), image quality assessment based on DFT spectrum analysis, Fourierbased restoration (e.g., deblurring), etc.

\subsection{Symmetrization}

A popular way to avoid side-effects due to the implicit periodization of the DFT is to apply a prior symmetrization to the image. The original discrete image $u: \Omega \rightarrow \mathbb{R}$ is first transformed into $u_{\sigma}: \Omega^{\prime} \rightarrow \mathbb{R}$, where

$$
\begin{aligned}
\forall(k, l) \in \Omega^{\prime}=\{0, \ldots, 2 M-1\} \times\{0, \ldots, 2 N-1\}, \quad u_{\sigma}(k, l)=u\left(\sigma_{M}(k), \sigma_{N}(l)\right), \\
\quad \text { and } \sigma_{M}(k)= \begin{cases}k & \text { if } \quad 0 \leq k \leq M-1, \\
2 M-1-k & \text { if } \quad M \leq k \leq 2 M-1\end{cases}
\end{aligned}
$$

(the "mirror" function $\sigma_{N}$ is defined similarly). Then, the DFT of $u_{\sigma}$ is considered. Since pixels from opposite borders of $\Omega^{\prime}$ are constrained to share the same gray level, the periodization of $u_{\sigma}$ does not create intensity gaps across the border of $\Omega^{\prime}$, so that the "cross structure" artifact is not present on $\widehat{u_{\sigma}}$ (see Figure 2). In the continuous domain, it can be seen that the prior 

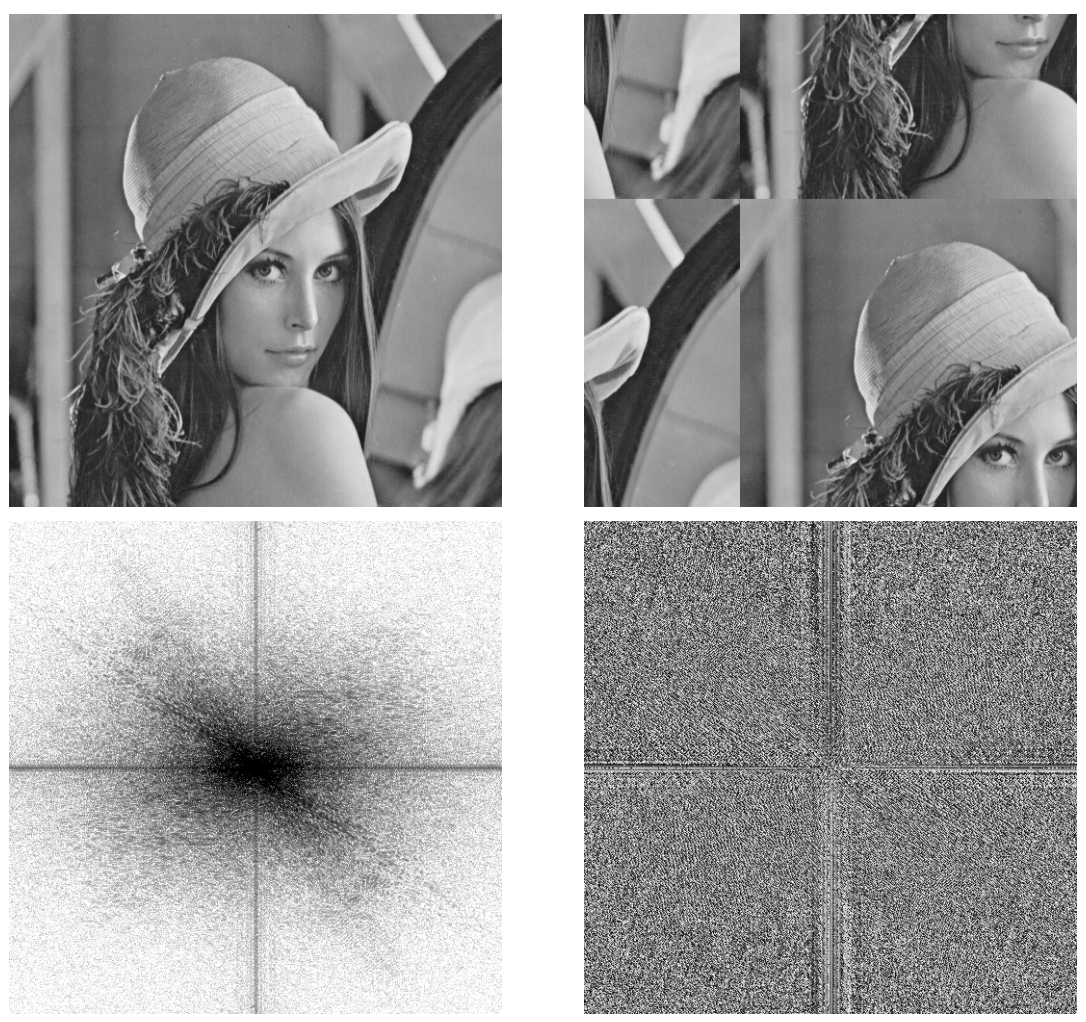

Figure 1: If the original Lena image (top, left) is assumed to be periodic as in the Discrete Fourier Transform framework, then it is equivalent to its translated version (top, right), which presents strong "discontinuities" across the original image frame border. These discontinuities create a "cross structure" artifact on its Discrete Fourier Transform, both for the modulus component (bottom, left, in log scale) and for the phase component (bottom, right).

symmetrization transforms the discontinuity of $u$ into a discontinuity of the normal derivative of $u_{\sigma}$, with much weaker consequences.

One apparent drawback of this symmetrization is that it multiplies by 4 the image size, so that a loss of computational efficiency could be expected. In fact, it can be easily shown that

$$
\widehat{u_{\sigma}}(q, r)=4 e^{i \pi q / 2 M} e^{i \pi r / 2 N} \cdot C_{u}(q, r)
$$

where

$$
C_{u}(q, r)=\sum_{(k, l) \in \Omega} \cos \left(\frac{\pi q}{M}\left(k+\frac{1}{2}\right)\right) \cos \left(\frac{\pi r}{N}\left(l+\frac{1}{2}\right)\right) u(k, l)
$$

is called the Discrete Cosine Transform (DCT) of $u$. Hence, the two-step process (symmetrization, then DFT) is simply equivalent to a DCT, followed by a linear phase shift (which corresponds in fact to a translation by $(1 / 2,1 / 2))$. Fast DCT algorithms are also available, and the amount of data to handle remains the same ( $M N$ real coefficients) thanks to the symmetry properties of $C_{u}$.

Contrary to window tapering methods (Hamming, etc.) mentioned in Section 1, prior symmetrization is well suited for image interpolation. For example, zero-padding image zooming 

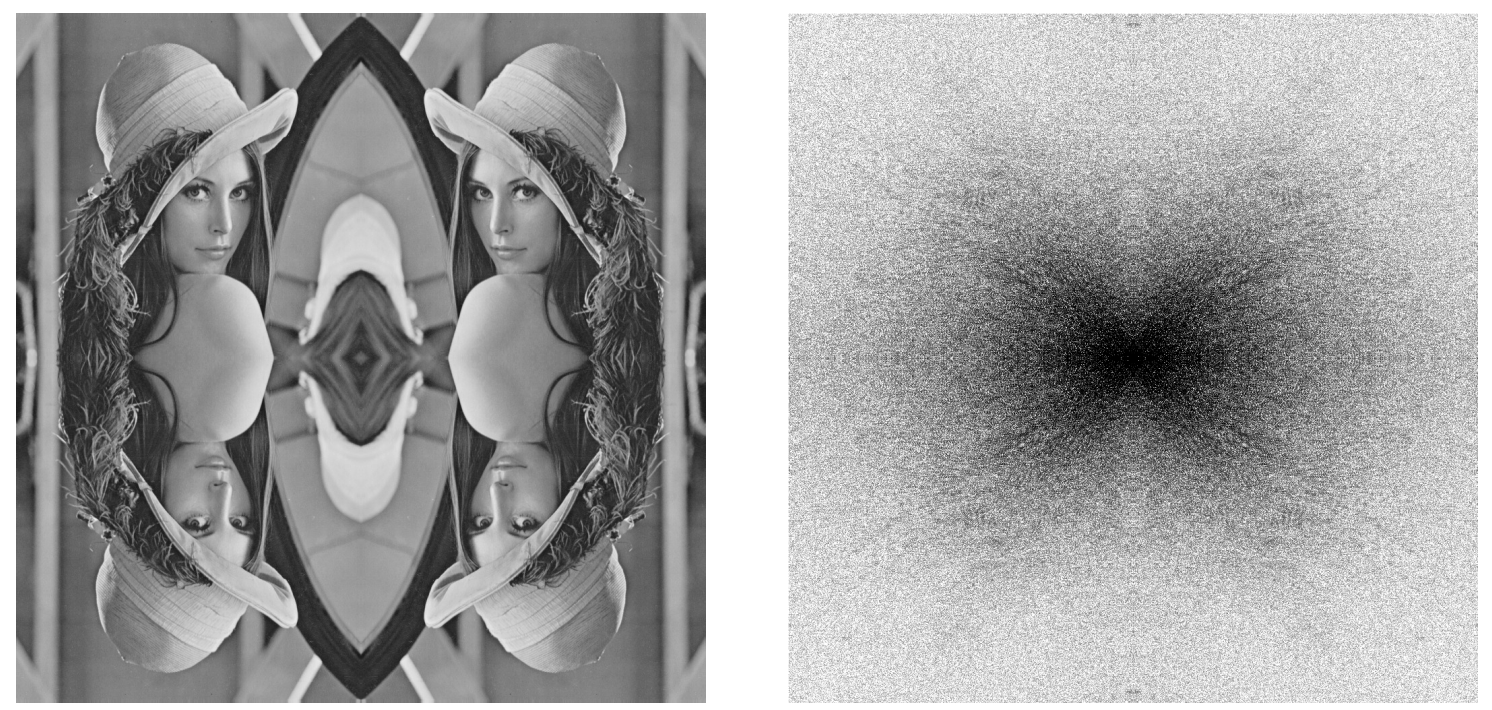

Figure 2: Symmetrization of the Lena image (left) and its corresponding Discrete Fourier Transform (modulus in log scale, right). Compared to Figure 1, the cross structure has disappeared in the Fourier domain, but the price to pay is a loss of information (orientation, phase component) due to the fact that the DFT of a symmetric image is constrained to be a symmetric real-valued function.

is easily performed through a DCT/inverse DCT process. Sub-pixel image translation is more heavy, since the resulting translated image has no reason to remain symmetric, which means that a more classical inverse DFT is necessary to reconstruct the translated image, which multiplies the memory and computation time requirements by a factor 4 .

But the most important drawback of prior symmetrization is the structure it induces on the DFT. Indeed, it is clear from (5) that $C_{u}$ is symmetric with respect to both axes, that is,

$$
\forall q, r, \quad C_{u}(q, r)=C_{u}(-q, r)=C_{u}(q,-r)=C_{u}(-q,-r) .
$$

This can be really annoying, in particular when image orientations issues are involved (for example, the orientation of a texture image cannot be properly analyzed with the DCT). Another property is that $C_{u}$ is a real-valued function, which means that its phase (argument) only takes the two values 0 and $\pi$ (modulo $2 \pi$ ). Possibilities of phase spectrum analysis are thus very limited on a symmetric image. In fact, the effect of prior symmetrization (followed by a DFT) is to transform $M N / 2$ complex coefficients into $M N$ real coefficients and $M N$ binary phase coefficients belonging to $\{0, \pi\}$, which may be, in terms of spectrum interpretation, a complete disaster.

Other symmetrization methods exist, in particular variants of (3) that do not duplicate boundary coefficients and transform a $M \times N$ image into a $(2 M-2) \times(2 N-2)$ image (or into a $(2 M-1) \times(2 N-1)$ image if only one side of the boundary is replicated). In all cases, the extended images are symmetric and the drawbacks mentioned above (orientation loss, phase binarization) still persist. Moreover, these methods may dramatically reduce the computational efficiency, since $M$ and $2 M-2$ cannot be both powers of two when $M>2$ (not to mention $2 M-1$ that can never be a power of two of course). 


\section{Periodic plus smooth decomposition}

\subsection{Definition}

As we saw, we have to face a dilemma. On the one hand, the Fast Fourier Transform is a very efficient algorithm that we would like to use for the global spectral analysis of discrete images and for image processing techniques admitting efficient implementations in Fourier domain. On the other hand the DFT causes strong artifacts due to the assumed periodicity of images. Here we propose to face this dilemma by using a decomposition of the discrete image $u$ into a sum of two images: an image $p$ (called "periodic component") that is well analyzed by the DFT and for most images visually very similar to $u$; and an image $s$ (called "smooth component") that has very smooth variations except at the borders of the image domain $\Omega$. There are several possibilities to define such a decomposition, but as we shall see later, the one we propose below has interesting theoretical and computational properties.

Theorem 1 (definition of periodic+smooth decomposition) Let $u \in \mathbb{R}^{\Omega}$ be a gray-level image, defined on a discrete rectangular domain $\Omega$. There exists a unique couple of images $(p, s) \in\left(\mathbb{R}^{\Omega}\right)^{2}$ that minimizes

$$
E(p, s)=\sum_{\substack{\mathbf{x} \in \Omega, \mathbf{y} \in \mathbb{Z}^{2} \backslash \Omega,|\mathbf{x}-\mathbf{y}|=1}}(p(\mathbf{x})-p(\dot{\mathbf{y}}))^{2}+\sum_{\substack{\mathbf{x} \in \Omega, \mathbf{y} \in \Omega,|\mathbf{x}-\mathbf{y}|=1}}(s(\mathbf{x})-s(\mathbf{y}))^{2}
$$

under the constraints

$$
u=p+s, \quad \text { and } \quad \operatorname{mean}(s)=0,
$$

where $\dot{\mathbf{y}}$ is the unique element of $\Omega$ equal to $\mathbf{y}$ modulo $\Omega$, and

$$
\operatorname{mean}(s)=\frac{1}{|\Omega|} \sum_{\mathbf{x} \in \Omega} s(\mathbf{x}) .
$$

The image $p$ is be called the periodic component of $u$ (also written $\operatorname{per}(u)$ ) and $s$ the smooth component of $u$.

Proof - Let us consider the following three minimization problems with respect to $s$ :

(1) Minimize $E(u-s, s)$ under the constraint mean $(s)=0$ (the minimization problem considered in the theorem);

(2) Minimize $F(s)$ under the constraint mean $(s)=0$, where

$$
F(s)=E(u-s, s)+\operatorname{mean}(s)^{2}
$$

(3) Minimize $F(s)$ without constraint.

Since the two functions $F(s)$ and $E(u-s, s)$ are equal under the constraint mean $(s)=0$, Problem (1) and (2) are equivalent. Now, if $s$ is a solution of Problem (3), then for any real number $\alpha$

$$
F(s+\alpha)=E(u-(s+\alpha), s+\alpha)+(\operatorname{mean}(s)+\alpha)^{2}=E(u-s, s)+(\operatorname{mean}(s)+\alpha)^{2},
$$


and since this should be minimum for $\alpha=0$, we necessarily have mean $(s)=0$. Hence Problem (2) and (3) are equivalent, and so are the initial Problem (1) and the Problem (3) we now consider.

First, we have

$$
F(s)=(u-s)^{T} Q_{1}(u-s)+s^{T} Q_{2} s,
$$

where $Q_{1}$ and $Q_{2}$ are positive symmetric linear mappings from $\mathbb{R}^{\Omega}$ into itself. Moreover, $Q_{2}$ is positive definite because

$$
s^{T} Q_{2} s=\sum_{\substack{\mathbf{x} \in \Omega, \mathbf{y} \in \Omega,|\mathbf{x}-\mathbf{y}|=1}}(s(\mathbf{x})-s(\mathbf{y}))^{2}+\operatorname{mean}(s)^{2}
$$

only vanishes when $s=0$. Hence, $Q_{1}+Q_{2}$ is invertible and from

$$
F(s)=u^{T} Q_{1} u-2 s^{T} Q_{1} u+s^{T}\left(Q_{1}+Q_{2}\right) s,
$$

we deduce that there exists a unique minimizer of $F$, given by

$$
\bar{s}=\left(Q_{1}+Q_{2}\right)^{-1} Q_{1} u .
$$

The "periodic plus smooth" decomposition we just defined is formulated in a variational setting, that imposes a compromise between the "good continuation" of $p$ across the boundary of $\Omega$ and the smoothness of $s$ inside the domain $\Omega$. The constraint $u=p+s$ ensures that an exact reconstruction of $u$ is possible from its two components while the other constraint mean $(s)=0$ forces $u$ and $p$ to have the same average gray value. In general, the linear decomposition of an image is questionable (contrary to sound signals for examples, images are not produced by the addition of waves but result from non-linear processes like occlusions), but since we are here interested in the Fourier spectrum of this image, the linear decomposition is very natural. Note, by the way, that the mapping $u \mapsto \operatorname{per}(u)$ is linear but of course not translation-invariant (since it has a special effects on the boundary of $\Omega$ ).

\subsection{Explicit computations}

A first interesting property of the "periodic plus smooth" decomposition is that it can be computed exactly in Fourier domain using only one additional DFT, as stated below.

Theorem 2 (computation in image-Fourier domain) Let $u \in \mathbb{R}^{\Omega}$ be a discrete $M \times N$ image, defined on $\Omega=\{0, \ldots, M-1\} \times\{0, \ldots, N-1\}$. Then we have

$$
\forall(q, r) \in \Omega \backslash(0,0), \quad \widehat{\operatorname{per}(u)}(q, r)=\hat{u}(q, r)-\frac{\hat{v}(q, r)}{2 \cos \left(\frac{2 \pi q}{M}\right)+2 \cos \left(\frac{2 \pi r}{N}\right)-4},
$$

and $\widehat{\operatorname{per}(u)}(0,0)=\hat{u}(0,0)$, where $v=v_{1}+v_{2}$ and

$$
\forall(x, y) \in \Omega, \quad v_{1}(x, y)=\left\{\begin{array}{l}
u(M-1-x, y)-u(x, y) \text { if } x=0 \text { or } x=M-1, \\
0 \text { else }
\end{array}\right.
$$




$$
\forall(x, y) \in \Omega, \quad v_{2}(x, y)=\left\{\begin{array}{l}
u(x, N-1-y)-u(x, y) \text { if } y=0 \text { or } y=N-1 \\
0 \text { else. }
\end{array}\right.
$$

Proof - Given a pixel $\mathbf{x} \in \Omega$, we decompose its 4 nearest neighbors modulo $\Omega$ into the sets

$$
\begin{gathered}
V(\mathbf{x})=\{\mathbf{y} \in \Omega,|\mathbf{x}-\mathbf{y}|=1\} \\
\text { and } W(\mathbf{x})=\left\{\mathbf{y} \in \Omega, \exists \mathbf{z} \in \mathbb{Z}^{2} \backslash \Omega, \dot{\mathbf{z}}=\mathbf{y} \text { and }|\mathbf{x}-\mathbf{z}|=1\right\} .
\end{gathered}
$$

Thus, $V(\mathbf{x})$ is the set of direct neighbors of $\mathbf{x}$, whereas $W(\mathbf{x})$ is the set of remaining neighbors arising from $\Omega$-periodization. Now from (6) and (8), we get

$$
F(s)=\sum_{\mathbf{x} \in \Omega}\left(\sum_{\mathbf{y} \in W(\mathbf{x})}(u(\mathbf{x})-s(\mathbf{x})-u(\mathbf{y})+s(\mathbf{y}))^{2}+\sum_{\mathbf{y} \in V(\mathbf{x})}(s(\mathbf{x})-s(\mathbf{y}))^{2}\right)+\operatorname{mean}(s)^{2} .
$$

If $s$ is the smooth component of $u$, then for all $\mathbf{x} \in \Omega$ the derivative of $F(s)$ with respect to $s(\mathbf{x})$ is zero, that is

$$
4 \sum_{\mathbf{y} \in W(\mathbf{x})}(-u(\mathbf{x})+s(\mathbf{x})+u(\mathbf{y})-s(\mathbf{y}))+4 \sum_{\mathbf{y} \in V(\mathbf{x})}(s(\mathbf{x})-s(\mathbf{y}))+\frac{2}{|\Omega|} \operatorname{mean}(s)=0 .
$$

Since $\operatorname{mean}(s)=0$, we obtain

$$
\forall \mathbf{x} \in \Omega, \quad \sum_{\mathbf{y} \in W(\mathbf{x})}(u(\mathbf{y})-u(\mathbf{x}))=-(|V(\mathbf{x})|+|W(\mathbf{x})|) s(\mathbf{x})+\sum_{\mathbf{y} \in V(\mathbf{x})} s(\mathbf{y})+\sum_{\mathbf{y} \in W(\mathbf{x})} s(\mathbf{y}) .
$$

Now $|V(\mathbf{x})|+|W(\mathbf{x})|=4$ for all $\mathbf{x} \in \Omega$, so we have

$$
B(u)=k \star s,
$$

where $\star$ represents the $\Omega$-periodic convolution operator, $k$ a convolution kernel defined by

$$
\forall \mathbf{x} \in \mathbb{Z}^{2}, \quad k(\mathbf{x})=\left\{\begin{array}{lll}
-4 & \text { if } & \mathbf{x}=\mathbf{0} \\
1 & \text { if } & |\mathbf{x}|=1 \\
0 & \text { else, }
\end{array}\right.
$$

and $B$ a linear operator defined by

$$
\forall \mathbf{x} \in \Omega, \quad B(u)(\mathbf{x})=\sum_{\mathbf{y} \in W(\mathbf{x})}(u(\mathbf{y})-u(\mathbf{x})) .
$$

One easily checks that $B(u)=v$, and that

$$
\forall(q, r) \in \mathbb{Z}^{2}, \quad \hat{k}(q, r)=2 \cos \left(\frac{2 \pi q}{M}\right)+2 \cos \left(\frac{2 \pi r}{N}\right)-4,
$$

so that by taking the Discrete Fourier Transform of (14), we obtain exactly the announced result (11) for $\operatorname{per}(u)=u-s$.

It seems surprising that the computation of the periodic component of $u$ can be done in Fourier domain, since the transform $u \mapsto \operatorname{per}(u)$ is precisely meant to be applied before a DFT to avoid artifacts. As we noticed before, the mapping $u \mapsto \operatorname{per}(u)$ is linear but not translationinvariant, so that it cannot be written as a periodic convolution and hence computed by a simple multiplication in Fourier domain. Nevertheless, the computation of $\operatorname{per}(u)$ can be done in four steps: 
1. compute the image $v$ to capture the intensity gaps of $u$ across the border of $\Omega$

2. compute the DFT of $v$

3. compute $\hat{s}$ with $(11)$, that is $\hat{s}(0,0)=0$ and

$$
\forall(q, r) \in \Omega \backslash(0,0), \quad \hat{s}(q, r)=\frac{\hat{v}(q, r)}{2 \cos \left(\frac{2 \pi q}{M}\right)+2 \cos \left(\frac{2 \pi r}{N}\right)-4}
$$

4. compute $u-\operatorname{per}(u)$ as the inverse DFT of $\hat{s}$, and finally $\operatorname{per}(u)$.

Steps 1 and 3 have a negligible complexity $(O(M+N)$ and $O(M N)$ respectively) compared to steps 2 and 4 , so that the effective cost of computing per $(u)$ is the cost of two DFTs.

We now give another interpretation of $\operatorname{per}(u)$, using the discrete Laplace operator.

Definition 1 (discrete Laplacian) Let $u: \Omega \rightarrow \mathbb{R}$ be a discrete $M \times N$ image. The discrete Laplacian of $u$ is the image $\Delta u: \Omega \rightarrow \mathbb{R}$ defined by

$$
\Delta u(\mathbf{x})=-4 u(\mathbf{x})+\sum_{\substack{\mathbf{y} \in \mathbb{Z}^{2} \\|\mathbf{x}-\mathbf{y}|=1}} u(\dot{\mathbf{y}})
$$

or equivalently in Fourier domain by

$$
\widehat{\Delta u}(q, r)=\left(2 \cos \left(\frac{2 \pi q}{M}\right)+2 \cos \left(\frac{2 \pi r}{N}\right)-4\right) \hat{u}(q, r) .
$$

We shall say that $u$ is harmonic on $S \subset \Omega$ if

$$
\forall \mathbf{x} \in S, \quad \Delta u(\mathbf{x})=0 .
$$

Using this definition, Equation (14) simply writes $\Delta s=B(u)$, so that the periodic component $p=\operatorname{per}(u)$ of an image $u$ is the unique solution of the discrete Poisson equation

$$
\Delta p=\Delta u-v,
$$

submitted to the constraint mean $(p)=$ mean $(u)$, where $v$ is the image defined in Theorem 2 . The classical way to solve Poisson equation is to use the standard DFT/FFT framework (see [6] for example), as we did above. In the case of (19), an interesting consequence is that one only needs one DFT to compute $\widehat{\operatorname{per}(u)}$, as shown by by the four-steps algorithm below:

1. compute the image $v$ to capture the intensity gaps of $u$ across the border of $\Omega$

2. compute the image $\Delta u$ by convolving $u$ with the kernel $k$ (Equation 15)

3. compute the Discrete Fourier Transform of $\Delta u-v$

4. compute $\widehat{\operatorname{per}(u)}$ using (19), that is

$$
\forall(q, r) \in \Omega \backslash(0,0), \quad \widehat{\operatorname{per}(u)}(q, r)=\frac{\widehat{\Delta u-v}(q, r)}{2 \cos \left(\frac{2 \pi q}{M}\right)+2 \cos \left(\frac{2 \pi r}{N}\right)-4} .
$$


Even if the simplest way to compute $\operatorname{per}(u)$ is to combine computations in the space domain and computations in the Fourier domain, as we did in the two algorithms above, it is interesting to understand the action of per in Fourier domain.

Proposition 1 (per in Fourier domain) If $u$ is a $M \times N$ discrete image, then for all $(q, r) \in$ $\Omega \backslash(0,0)$ one has

$$
\begin{gathered}
\widehat{\operatorname{per}(u)}(q, r)=\hat{u}(q, r)+\varphi(q, r)^{-1}\left(\psi_{M}(q) \sum_{a=0}^{M-1} \psi_{M}(-a) \hat{u}(a, r)+\psi_{N}(r) \sum_{b=0}^{N-1} \psi_{N}(-b) \hat{u}(q, b)\right), \\
\text { where } \psi_{n}(t)=\frac{e^{2 i \pi t / n}-1}{\sqrt{n}} \text { and } \varphi(q, r)=2 \cos \left(\frac{2 \pi q}{M}\right)+2 \cos \left(\frac{2 \pi r}{N}\right)-4 .
\end{gathered}
$$

Proof - We start from

$$
\begin{aligned}
\widehat{v}_{1}(q, r) & =\sum_{x=0}^{M-1} \sum_{y=0}^{N-1} v_{1}(x, y) \exp \left[-2 i \pi\left(\frac{q x}{M}+\frac{r y}{N}\right)\right] . \\
& =\left(e^{2 i \pi q / M}-1\right) \cdot \sum_{y=0}^{N-1}(u(0, y)-u(M-1, y)) \cdot e^{-2 i \pi r y / N} .
\end{aligned}
$$

Then, using the Inverse DFT we obtain

$$
\begin{aligned}
\sum_{y=0}^{N-1} u(0, y) \cdot e^{-2 i \pi r y / N} & =\sum_{y=0}^{N-1} e^{-2 i \pi r y / N} \frac{1}{M N} \sum_{s=0}^{M-1} \sum_{t=0}^{N-1} \hat{u}(s, t) e^{2 i \pi t y / N} \\
& =\frac{1}{M N} \sum_{s=0}^{M-1} \sum_{t=0}^{N-1} \hat{u}(s, t) \sum_{y=0}^{N-1} e^{2 i \pi(t-r) y / N} \\
& =\frac{1}{M} \sum_{s=0}^{M-1} \hat{u}(s, r) .
\end{aligned}
$$

Similarly,

$$
\sum_{y=0}^{N-1} u(M-1, y) \cdot e^{-2 i \pi r y / N}=\frac{1}{M} \sum_{s=0}^{M-1} \hat{u}(s, r) e^{-2 i \pi s / M},
$$

so that in the end

$$
\widehat{v_{1}}(q, r)=\frac{e^{2 i \pi q / M}-1}{M} \cdot \sum_{s=0}^{M-1} \hat{u}(s, r) \cdot\left(1-e^{-2 i \pi s / M}\right) .
$$

By symmetry,

$$
\widehat{v_{2}}(q, r)=\frac{e^{2 i \pi r / N}-1}{N} \cdot \sum_{t=0}^{N-1} \hat{u}(q, t) \cdot\left(1-e^{-2 i \pi t / N}\right),
$$

and the announced formula directly results from (11). 
An interesting property that results from Proposition 1 is that in Fourier domain, the per operator is a linear filter whose support has a "cross" shape, as the value of $\widehat{\operatorname{per}(u)}(q, r)$ only depends on the values of $\hat{u}(q, \cdot)$ and $\hat{u}(\cdot, r)$. Nonetheless, the direct estimation of $\widehat{\operatorname{per}(u)}$ in Fourier domain using Proposition 1 is not computationally efficient in general since it requires (in addition to the computation of $\hat{u}) O((M+N) M N)$ operations, versus $O(M N \log (M N))$ operations for the estimate of $\hat{v}$ and (11) when $M$ and $N$ are powers of two.

We now give an explicit form for the per operator in the image domain.

Proposition 2 (per in image domain) If $u$ is a $M \times N$ discrete image, then for all pixels $\left(x^{\prime}, y^{\prime}\right) \in \Omega$ we have

$$
\begin{aligned}
\operatorname{per}(u)\left(x^{\prime}, y^{\prime}\right)=u\left(x^{\prime}, y^{\prime}\right) & -\sum_{x=0}^{M-1}(u(x, 0)-u(x, N-1)) \cdot\left(\gamma\left(x^{\prime}-x, y^{\prime}\right)-\gamma\left(x^{\prime}-x, y^{\prime}+1\right)\right) \\
& -\sum_{y=0}^{N-1}(u(0, y)-u(M-1, y)) \cdot\left(\gamma\left(x^{\prime}, y^{\prime}-y\right)-\gamma\left(x^{\prime}+1, y^{\prime}-y\right)\right),
\end{aligned}
$$

where the DFT of the $(M, N)$-periodic image $\gamma$ satisfies $\widehat{\gamma}(0,0)=0$ and

$$
\forall(q, r) \in \Omega \backslash\{0\}, \quad \widehat{\gamma}(q, r)=\frac{1}{2 \cos \left(\frac{2 \pi q}{M}\right)+2 \cos \left(\frac{2 \pi r}{N}\right)-4} .
$$

Proof - By taking the Inverse DFT in (11), we obtain

$$
\forall\left(x^{\prime}, y^{\prime}\right) \in \Omega, \quad \operatorname{per}(u)\left(x^{\prime}, y^{\prime}\right)=u\left(x^{\prime}, y^{\prime}\right)-(v \star \gamma)\left(x^{\prime}, y^{\prime}\right)
$$

as $\widehat{v \star \gamma}=\hat{v} \cdot \hat{\gamma}$. Hence,

$$
\begin{aligned}
\operatorname{per}(u)\left(x^{\prime}, y^{\prime}\right)= & u\left(x^{\prime}, y^{\prime}\right) \\
= & -\sum_{x=0}^{M-1} \sum_{y=0}^{N-1} v(x, y) \gamma\left(x^{\prime}-x, y^{\prime}-y\right) \\
= & -\sum_{x=0}^{M-1} v_{2}(x, 0) \gamma\left(x^{\prime}-x, y^{\prime}\right)+v_{2}(x, N-1) \gamma\left(x^{\prime}-x, y^{\prime}-N+1\right) \\
& -\sum_{y=0}^{N-1} v_{1}(0, y) \gamma\left(x^{\prime}, y^{\prime}-y\right)+v_{1}(M-1, y) \gamma\left(x^{\prime}-M+1, y^{\prime}-y\right)
\end{aligned}
$$

and the announced result follows from the expressions of $v_{1}$ and $v_{2}$ (see Theorem 2) and the fact that $\gamma$ is $(M, N)$-periodic.

Note that on the space of zero-mean images, the application $u \mapsto \gamma \star u$ is the inverse of the Laplace operator, that is $\gamma \star u=\Delta^{-1} u$ (to be compared to $k \star u=\Delta u$ ).

Contrary to Proposition 1 that was not computationally efficient compared to (11), Proposition 2 can be useful if the periodic component has to be evaluated only in a small sub-domain of $\Omega$. Indeed, since the $\gamma$ function (that only depends on $M$ and $N$ ) can be computed in advance, the evaluation of $\operatorname{per}(u)$ in a single point $\left(x^{\prime}, y^{\prime}\right)$ of $\Omega$ can be realized with Proposition 2 using only $O(M+N)$ operations. 


\subsection{Properties of the periodic component}

In this part, we investigate several properties of the periodic+smooth decomposition. For an image domain $\Omega=\{0, \ldots, M-1\} \times\{0, \ldots, N-1\}$, we shall write

$$
\stackrel{\Omega}{\Omega}=\{1, \ldots, M-2\} \times\{1, \ldots, N-2\} \quad \text { and } \quad \partial \Omega=\Omega \backslash \stackrel{\Omega}{ } .
$$

Definition 2 (p-connected image) We say that an image $u: \Omega \rightarrow \mathbb{R}$ is p-connected (the letter $p$ refers to the periodization process) if

$$
\forall \mathbf{x} \in \partial \Omega, \quad \forall \mathbf{y} \in W(\mathbf{x}), \quad u(\mathbf{y})=u(\mathbf{x}),
$$

where $W(\mathbf{x})$ is defined in (13). We denote by $\mathcal{P}$ the vector space of p-connected images.

Hence, a p-connected image is an image for which all intensity gaps arising across the border of $\Omega$ are zero. Notice that for any image $u$, the symmetrized image $u_{\sigma}$ (defined in Section 2.2) is p-connected.

Proposition 3 The dimension of the space $\mathcal{P}$ of $p$-connected $M \times N$ images is

$$
\operatorname{dim} \mathcal{P}=M N-M-N+1 .
$$

Proof - Let us write $\left(\delta_{(k, l)}\right)_{(k, l) \in \Omega}$ the canonical basis (discrete Dirac basis) of $\mathbb{R}^{\Omega}$. We define several sequences of images:

- a sequence $b_{I}$ (inside points) of $(M-2)(N-2)$ images, the canonical basis of $\Omega$ defined by the images

$$
\delta_{\mathbf{x}}, \quad \mathbf{x} \in\{1 . . M-2\} \times\{1 . . N-2\} .
$$

- a sequence $b_{E}$ (edge points) of $M+N-4$ images of $\partial \Omega$ defined by

$$
\delta_{\mathbf{x}}+\delta_{w(\mathbf{x})}, \quad \mathbf{x} \in(\{1 . . M-2\} \times\{0\}) \cup(\{0\} \times\{1 . . N-2\}),
$$

where $w(\mathbf{x})$ is the unique element of $W(\mathbf{x})$.

- four images $c_{1}, c_{2}, c_{3}, c_{4}$ (corners) defined by

$$
\left(\begin{array}{c}
c_{1} \\
c_{2} \\
c_{3} \\
c_{4}
\end{array}\right)=P\left(\begin{array}{c}
\delta_{(0,0)} \\
\delta_{(M-1,0)} \\
\delta_{(0, N-1)} \\
\delta_{(M-1, N-1)}
\end{array}\right), \quad \text { with } \quad P=\left(\begin{array}{cccc}
1 & 1 & 1 & 1 \\
1 & 1 & -1 & -1 \\
1 & -1 & 1 & -1 \\
1 & -1 & -1 & 1
\end{array}\right) \text {. }
$$

- a sequence $b_{G}$ (edge gaps) of $M+N-4$ images of $\partial \Omega$ defined by

$$
\delta_{\mathbf{x}}-\delta_{w(\mathbf{x})}, \quad \mathbf{x} \in(\{1 . . M-2\} \times\{0\}) \cup(\{0\} \times\{1 . . N-2\}) .
$$


The sequence obtained by concatenation of the sequences $b_{I}, b_{E}, c_{1}, c_{2}, c_{3}, c_{4}$ and $b_{G}$ is clearly orthogonal (in particular because the matrix $P$ is orthogonal), and its length is

$$
(M-2)(N-2)+M+N-4+4+M+N-4=M N,
$$

hence it is an orthogonal basis of $\mathbb{R}^{\Omega}$. Among these images, the first $M N-M-N+1$ ones $\left(b_{I}\right.$, $\left.b_{E}, c_{1}\right)$ belong to $\mathcal{P}$, while the remaining $M+N-1$ ones $\left(c_{2}, c_{3}, c_{4}, b_{G}\right)$ belong to $\mathcal{P}^{\perp}$, which achieves the proof.

Theorem 3 (properties of the per operator) Let $u: \Omega \rightarrow \mathbb{R}$ be a discrete image. Then we have the following properties:

( $i) \quad u-\operatorname{per}(u)$ only depends on $\left.u\right|_{\partial \Omega}$

(ii) $u-\operatorname{per}(u)$ is harmonic on $\Omega$

(iii) per is an isomorphism of $\mathbb{R}^{\Omega}$

(iv) all complex eigenvalues of per are real and belong to $(0,1]$

$(v) \operatorname{ker}($ per $-i d)=\mathcal{P}$

(vi) per is diagonalizable.

Proof - Property $(i)$ is a direct consequence of Theorem 2, which shows that $u$ - $\operatorname{per}(u)$ only depends on $v$, and $v$ itself only depends on the values taken by $u$ on $\partial \Omega$. As for Property (ii), it follows from (14), (16) and the fact that $\Delta u=k \star u$, since for any $\mathbf{x} \in \stackrel{\Omega}{ }$ we have $W(\mathbf{x})=\emptyset$ and consequently $B(u)(\mathbf{x})=0$. To show that per is an isomorphism of $\mathbb{R}^{\Omega}$ (that is, an invertible linear mapping from $\mathbb{R}^{\Omega}$ onto itself), we first deduce from (10) that

$$
\operatorname{per}(u)=u-s=u-\left(Q_{1}+Q_{2}\right)^{-1} Q_{1} u=\left(Q_{1}+Q_{2}\right)^{-1} Q_{2} u,
$$

and we conclude by noticing that $Q_{2}$ is invertible (it is positive definite).

Now let us prove that all complex eigenvalues of per belong to $(0,1]$ (Property $(i v)$ ). Let $\lambda \in \mathbb{C}$ be a complex eigenvalue of per, associated to a complex eigenvector $u \in \mathbb{C}^{\Omega} \backslash\{0\}$. From (20) and $\operatorname{per}(u)=\lambda u$, we deduce that

$$
\left(Q_{1}+Q_{2}\right)(\lambda u)=Q_{2} u
$$

so that if we multiply both sides by $u^{*}$ (the conjugate transpose of $u$ ), we obtain

$$
\lambda u^{*}\left(Q_{1}+Q_{2}\right) u=u^{*} Q_{2} u .
$$

Since $u^{*} Q_{2} u>0\left(Q_{2}\right.$ definite positive hermitian $)$ and $u^{*} Q_{1} u \geq 0\left(Q_{1}\right.$ non-negative hermitian), we obtain as desired that

$$
\lambda=\frac{u^{*} Q_{2} u}{u^{*} Q_{1} u+u^{*} Q_{2} u} \in(0,1] .
$$

Let us find the eigenspace associated to the eigenvalue 1. Using (20), we get

$$
\operatorname{ker}(\operatorname{per}-i d)=\operatorname{ker}\left(\left(Q_{1}+Q_{2}\right)^{-1} Q_{2}-i d\right)=\operatorname{ker}\left(-\left(Q_{1}+Q_{2}\right)^{-1} Q_{1}\right)=\operatorname{ker} Q_{1} .
$$


Now from (6), (8) and (9) we obtain

$$
p^{T} Q_{1} p=\sum_{\substack{\mathbf{x} \in \Omega, \mathbf{y} \in \mathbb{Z}^{2} \backslash \Omega,|\mathbf{x}-\mathbf{y}|=1}}(p(\mathbf{x})-p(\dot{\mathbf{y}}))^{2}=\sum_{\mathbf{x} \in \Omega} \sum_{\mathbf{y} \in W(\mathbf{x})}(p(\mathbf{x})-p(\mathbf{y}))^{2},
$$

and this sum vanishes if and only if all terms are zero. Hence,

$$
p \in \operatorname{ker}(\text { per }-i d) \Leftrightarrow p \in \operatorname{ker} Q_{1} \Leftrightarrow \forall \mathbf{x} \in \Omega, \forall \mathbf{y} \in W(\mathbf{x}), p(\mathbf{x})=p(\mathbf{y}) \Leftrightarrow p \in \mathcal{P},
$$

which proves Property $(v)$.

Last, we show that per is diagonalizable. Since $Q_{1}+Q_{2}$ and $Q_{2}$ are symmetric positive definite matrices, there exists an invertible matrix $P$ such that

$$
P^{T}\left(Q_{1}+Q_{2}\right) P=I \quad \text { and } \quad P^{T} Q_{2} P=D,
$$

where $I$ is the identity matrix and $D$ a diagonal matrix (such a matrix $P$ is obtained by considering, among all bases that are orthonormal for the inner product defined by $Q_{1}+Q_{2}$, one that is orthogonal for the inner product defined by $Q_{2}$ ). Now if $A$ is the matrix of per in the canonical basis of $\mathbb{R}^{\Omega}$, by (20) we have $\left(Q_{1}+Q_{2}\right) A=Q_{2}$ so that

$$
P^{-1} A P=P^{T}\left(Q_{1}+Q_{2}\right) P P^{-1} A P=P^{T}\left(Q_{1}+Q_{2}\right) A P=P^{T} Q_{2} P=D,
$$

which proves that $P$ diagonalizes $A$.

Property (ii) is an interesting guarantee that $\operatorname{per}(u)$ and $u$ behave the same way locally. In particular, they have the same discrete Laplacian on $\Omega$, so that according to Marr and Hildreth, who proposed an edge-detection theory based on the zero-crossings of the Laplacian [13], $u$ and $\operatorname{per}(u)$ have the same contours.

Concerning the spectrum of per (written $\Sigma(M, N)$ ), Property (iv) shows that $\Sigma(M, N) \subset$ $(0,1]$ (which guarantees in particular the stability of per with respect to noise) but it is worth mentioning that more precise estimates could probably be obtained. Indeed, numerical computations seem to indicate that

$$
\forall M, N, \quad \min \Sigma(M, N)=\min \left(\frac{1}{M}, \frac{1}{N}\right),
$$

and that for all square images

$$
\forall N, \quad \max (\Sigma(N, N) \backslash\{1\})<\frac{1}{\sqrt{2}}
$$

(the bound seeming a little higher than $1 / \sqrt{2}$ for non-square images).

Property (v) tells us that the images that are invariant under per are exactly the p-connected images. Thus, it is natural to wonder if the iteration of the per operator permits to define a projection on $\mathcal{P}$. As we see now, the answer is yes, thanks to Property (iv).

Proposition 4 (the projector $p^{\infty}$ ) Let us write per $^{n}$ the operator obtained by iterating $n$ times per, that is,

$$
\operatorname{per}^{n}=\text { per } \circ \text { per } \ldots \circ \text { per } .
$$

Then for any discrete image $u$, the sequence of images $\operatorname{per}^{n}(u)$ converges to an image denoted by $\operatorname{per}^{\infty}(u)$, and $\operatorname{per}^{\infty}$ is a linear projection from $\mathbb{R}^{\Omega}$ onto $\mathcal{P}$. 
Proof - Since per is diagonalizable, we can build a basis $b$ of $\mathbb{R}^{\Omega}$ whose first $M+N-1$ vectors are eigenvectors of per associated to eigenvalues in $(0,1)$, while the remaining vectors form a basis of $\mathcal{P}=\operatorname{ker}($ per $-i d)$. Then, the matrix of per $^{n}$ in this basis is simply

$$
\operatorname{Mat}_{b}\left(\operatorname{per}^{n}\right)=\left(\begin{array}{cc}
D^{n} & 0 \\
0 & I
\end{array}\right)
$$

where $I$ is the identity matrix and $D$ is a diagonal matrix whose elements belong to $(0,1)$. Since $D^{n} \rightarrow 0$ as $n \rightarrow \infty$, we conclude that per $^{n}$ converges as $n \rightarrow \infty$ to a linear operator per $^{\infty}$ defined by

$$
\operatorname{Mat}_{b}\left(\operatorname{per}^{\infty}\right)=\left(\begin{array}{cc}
0 & 0 \\
0 & I
\end{array}\right)
$$

Hence, per $^{\infty}$ is a linear projection whose image is $\mathcal{P}$.

A natural question that arises after Proposition 4 is the speed of convergence. Indeed, the convergence is geometric, and is mainly governed by the maximum non-1 eigenvalue of per. It appears from the conjectured bound (22) that we can expect a good convergence in a dozen of iterations or so, since

$$
\left(\frac{1}{\sqrt{2}}\right)^{12}=2^{-6}=\frac{1}{64}
$$

which is an acceptable numerical precision for most image applications.

Now a more subtle question is: Should we iterate per or not? We do not have a definitive answer to this question, especially because as we just saw the per ${ }^{\infty}$ projection can be quickly evaluated. However, we can argue that even periodic images (that is, synthetic images build in a periodic setting) do not belong to $\mathcal{P}$ in general. Indeed, for a "periodic" image we should expect the intensity gaps across the frame borders to have the same order of magnitude than the gaps between adjacent pixels inside the image domain. As we shall see in Section 3.4, this is more or less the case for $\operatorname{per}(u)$, but clearly not the case for per ${ }^{\infty}$ (for which all outside gaps are zero). Another way to say this is: after translating (with periodicity) a periodic image, we should not be able, with local inspection, to determine where the original frame border was. In $\operatorname{per}^{\infty}(u)$, this is clearly false, since this image has two identical adjacent lines and two identical adjacent columns. Hence, it seems more reasonable in general to transform an image $u$ into $\operatorname{per}(u)$, which is close to $\mathcal{P}$, than into $\operatorname{per}^{\infty}(u)$, which belongs to $\mathcal{P}$.

If, for some reason, a projection onto $\mathcal{P}$ is desirable, then per ${ }^{\infty}$ is an interesting solution, unlike the canonical orthogonal $\left(l^{2}\right)$ projection $\pi$ defined by

$$
\|\pi(u)-u\|_{2}=\inf _{v \in \mathcal{P}}\|v-u\|_{2}
$$

where as usual $\|u\|_{2}^{2}=\sum_{\mathbf{x} \in \Omega} u(\mathbf{x})^{2}$. Indeed, it is very easy to see that $\pi(u)$ and $u$ are equal on $\stackrel{\Omega}{\Omega}$, which means that the intensity gaps across the boundary of $\Omega$ are simply moved by $\pi$ into inside intensity gaps between $\partial \Omega$ and $\Omega$.

\subsection{The one-dimensional case}

In this section, we consider the one-dimensional case $(M=1, N>1)$, which boils down to applying the periodic plus smooth decomposition to a signal $u:\{0, \ldots, N-1\} \rightarrow \mathbb{R}$. The 
interest of this part is not to bring a new remedy to edge effects for signals (the problem is much simpler than for images, and does not require all that precedes), but simply to give simple explicit formulations of the periodic plus smooth decomposition and the per ${ }^{\infty}$ projection in this case.

Proposition 5 The periodic component of a 1 -D signal $u:\{0, \ldots N-1\} \rightarrow \mathbb{R}$ is given by

$$
\forall k \in\{0, \ldots N-1\}, \quad \operatorname{per}(u)(k)=u(k)-\frac{u(N-1)-u(0)}{N} \cdot\left(k-\frac{N-1}{2}\right) .
$$

Proof - We derive an elementary proof in order this part to be self-contained. If $s$ is the smooth component of $u$, according to (8) it minimizes

$$
F(s)=(u(0)-s(0)-u(N-1)+s(N-1))^{2}+\sum_{k=0}^{N-2}(s(k+1)-s(k))^{2}+\left(\frac{1}{N} \sum_{k=0}^{N-1} s(k)\right)^{2},
$$

(the last term being 0 ), and the Euler equations $\frac{\partial F(s)}{\partial s(k)}=0$ lead to

$$
\forall k \in\{1, \ldots N-2\}, \quad s(k)-s(k-1)-(s(k+1)-s(k))=0,
$$

and

$$
\left\{\begin{array}{l}
-(u(0)-s(0)-u(N-1)+s(N-1))-(s(1)-s(0))=0, \\
\quad u(0)-s(0)-u(N-1)+s(N-1)+s(N-1)-s(N-2)=0 .
\end{array}\right.
$$

Writing $a=s(1)-s(0)$, we obtain from (25) that

$$
s(k+1)-s(k)=s(k)-s(k-1)=\ldots=s(1)-s(0)=a,
$$

so that $s$ is an affine function (that is, harmonic). Since it has zero mean, we have

$$
\forall k \in\{0, \ldots N-1\}, \quad s(k)=a \cdot\left(k-\frac{N-1}{2}\right),
$$

and the unknown coefficient $a$ satisfies, thanks to the two equivalent equations in (26),

$$
u(0)-u(N-1)+a(N-1)+a=0 .
$$

Equation (24) then simply follows from $\operatorname{per}(u)=u-s$.

Proposition 6 Let $u:\{0, \ldots N-1\} \rightarrow \mathbb{R}$ be a $1-D$ signal. Then

$$
\forall k \in\{0, \ldots N-1\}, \quad \operatorname{per}^{\infty}(u)(k)=u(k)-\frac{u(N-1)-u(0)}{N-1} \cdot\left(k-\frac{N-1}{2}\right) .
$$

Proof - Thanks to Proposition 5, we have $\operatorname{per}(u)=u-a \cdot L$ with

$$
a=\frac{u(N-1)-u(0)}{N} \quad \text { and } \quad \forall k \in\{0, \ldots N-1\}, \quad L(k)=k-\frac{N-1}{2} .
$$


Since $\operatorname{per}(L)=N^{-1} L$, we have $\operatorname{per}^{m}(L)=N^{-m} L$ for any positive integer $m$ and as a consequence

$$
\operatorname{per}^{m+1}(u)=\operatorname{per}^{m}(u)-a N^{-m} L .
$$

Summing (28) from $m=0$ to $m=n-1$ (for some positive integer $n$ ) yields

$$
\operatorname{per}^{n}(u)=u-a\left(\sum_{m=0}^{N-1} N^{-m}\right) L=u-a \frac{1-N^{-m}}{1-N^{-1}} L \underset{n \rightarrow+\infty}{\longrightarrow} u-a \frac{N}{N-1} L,
$$

which corresponds exactly to (27).

As we can observe, both per and per ${ }^{\infty}$ consist, for a one-dimensional signal $u$, in the subtraction of a ramp signal depending only on the value of the gap $u(N-1)-u(0)$. The difference between per and per ${ }^{\infty}$ is that per $^{\infty}$ forces the equality between endpoint values (zero gap), while per merely divides the original gap by $N$.

For two-dimensional images, the situation is more complex (and interesting) since the space of harmonic images is much wider than the space of harmonic signals (which boils down to affine signals). This offers a greater flexibility for the periodic plus smooth decomposition of images and explains, as we shall see now, why the periodic component of an image manages to avoid DFT artifacts while preserving in most cases the visual appearance of the original image.

\subsection{Comparison with the Polyharmonic Local Cosine and Sine Transforms}

There is some connection between the periodic plus smooth decomposition we propose here and the Polyharmonic Transforms defined by Saito and his collaborators.

Let us recall that given a discrete image $u: \Omega \rightarrow \mathbb{R}$, its smooth component $s=u-\operatorname{per}(u)$ can be viewed as the unique solution of Poisson's Equation

$$
\begin{cases}\Delta s=0 & \text { in } \stackrel{\Omega}{ } \\ \Delta s=B(u) & \text { on } \partial \Omega \\ \operatorname{mean}(s)=0, & \end{cases}
$$

where $B(u)$ is the "gap" of $u$ accross $\partial \Omega$ (see Equation 16).

The Polyharmonic Local Sine Transform PHLST) was introduced by Saito and Remy [22], and then improved by Zhao, Saito and Wang [28]. Keeping the same notations as before, it consists also in decomposing an image $u$ under the form $u=p+s$, where, in the simplest (order 1) version, the residual image $s$ satisfies

$$
\begin{cases}\Delta s=0 & \text { in } \AA \\ s=u & \text { on } \partial \Omega\end{cases}
$$

(we kept the notation of the present paper to make the comparison easier). As we can see, the difference with the periodic plus smooth decomposition relies in the boundary condition, that forces the component $p=u-s$ of the PHLST to vanish on $\partial \Omega$. This decomposition hence leads to images $p$ that are in general not as close to the initial image $u$ as is $\operatorname{per}(u)$ (recall in particular that one has $\operatorname{per}(u)=u$ as soon as $u \in \mathcal{P})$. 
Another decomposition, the Polyharmonic Local Cosine Transform (PHLCT), was introduced by Yamatani and Saito in [27]. Again, the residual image satisfies a Poisson-type Equation, that can be written, in a continuous setting,

$$
\begin{cases}\Delta s=K & \text { in } \AA \\ \frac{\partial s}{\partial \nu}=\frac{\partial u}{\partial \nu} & \text { on } \partial \Omega, \\ \operatorname{mean}(s)=0, & \end{cases}
$$

where $\frac{\partial u}{\partial \nu}$ denotes the normal derivative of $u$ (defined on the boundary of $\Omega$ ) and $K$ is a constant equal to the average normal derivative of $u$ along the boundary of $\Omega$. The interest of this decomposition is that the normal derivative of $p=u-s$ vanishes along the boundary of $\Omega$, so that after symmetrization (that is implicit with the DCT) and periodization the regularity of $p$ across the boundary is improved. But the PHLCT is not a good "periodic component" as such, since it requires a symmetrization process to avoid periodization artifacts (see Section 2.2).

\section{Applications}

In this part, we discuss the interest of the periodic plus smooth decomposition in several image processing issues.

\subsection{Spectrum analysis}

The decomposition of an image $u$ into its periodic component $p=\operatorname{per}(u)$ and its smooth component $s=u-p$ was proposed in Section 3.1 as an attempt to avoid DFT artifacts (the "cross structure" shown on Figure 1). The idea is that since real-world images are not periodic, they should be decomposed into the sum of a periodic component $(p)$, whose spectrum is close to the spectrum of $u$ but artifact-free, and a smooth component capturing (almost) all edge effects arising from the periodization of $u$. On Figure 3, we can see that the $p+s$ decomposition manages rather well to achieve this task. Not only the periodic component $p$ is visually very close to $u$ (the difference lying in what appear to be slow contrast changes), but the "cross artifact" completely disappears in the Fourier transform of $p$, while the other parts of the spectrum seems unchanged. The decomposition of the "boundary discontinuities" of the original image is shown on Figure 4, where the three images $u, p$ and $s$ have been replicated in a periodic manner. Other examples of periodic components are given on Figure 5.

\subsection{Shannon interpolation}

According to Shannon Theory, a well-sampled bandlimited image can be exactly reconstructed with cardinal sine (sinc) interpolation. This applies for images defined on the whole plane $\mathbb{R}^{2}$, but also for periodic images, the latter case being particularly interesting because periodic sinc interpolation corresponds to zero-padding in the Fourier domain, which can be realized efficiently using the FFT. However, periodic sinc interpolation often causes strong ringing artifacts on the frame borders, so that a symmetrization process (see Section 2.2) is generally used, which substantially increases time and memory requirements. 

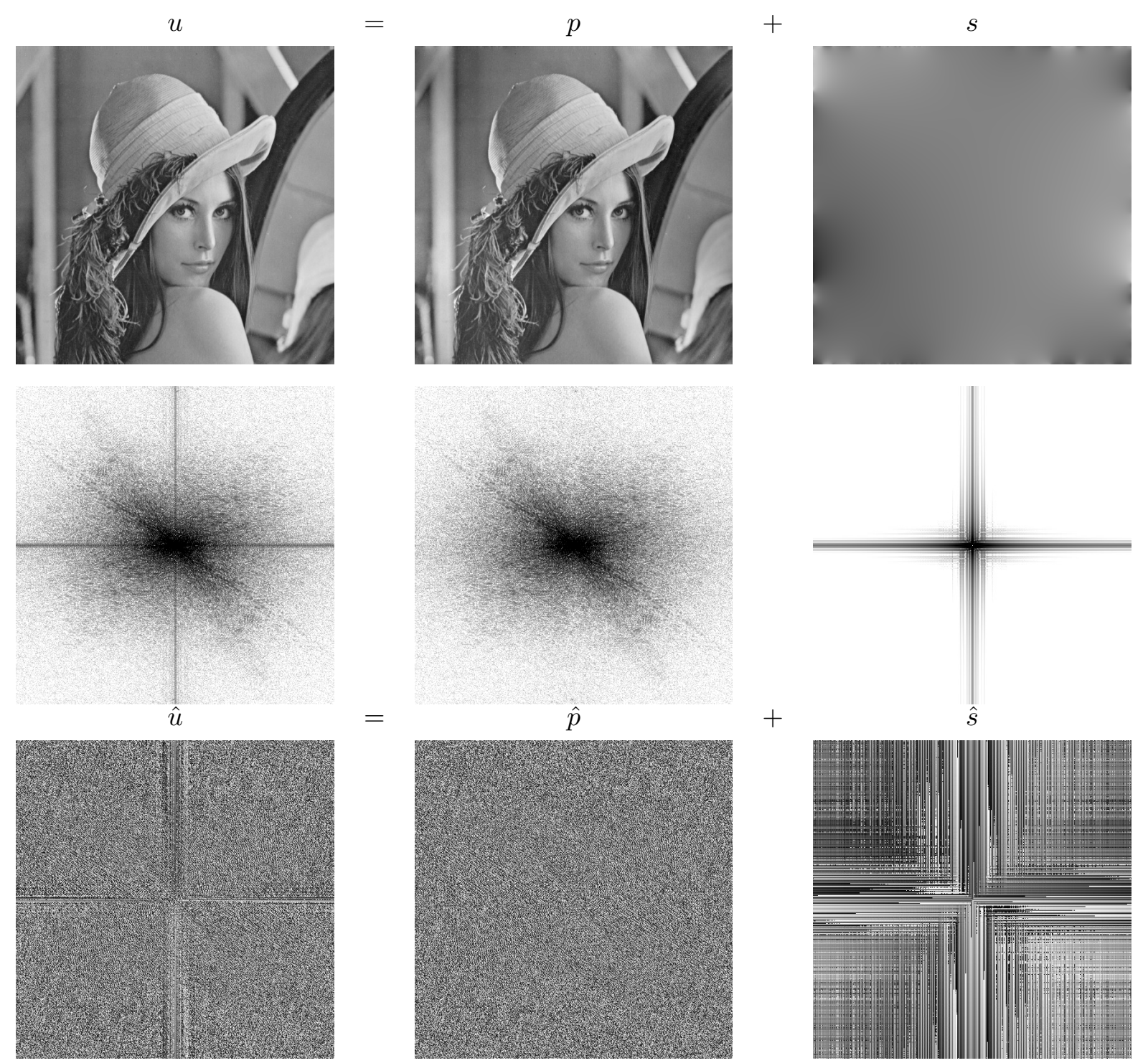

Figure 3: The original Lena image $u$ (top row, left column) is decomposed into its periodic component $p$ (top row, middle column) and its smooth component $s$ (top row, right column). The Fourier spectrum of each of these three images is represented underneath: the modulus in log scale appears on the middle row, and the argument (phase) on the bottom row. As we can see, the periodic component captures not only most of the visual information (the difference between $u$ and $p$ being noticeable only at large scales), but also the relevant data for DFT purposes, allowing a pleasant artifact-free restoration of the spectrum of $u$. On the contrary, the smooth component mainly gather the frame border discontinuities in the image domain, and equivalently the consequent "cross structure" in the Fourier domain. Note that the grey levels of the smooth image have been shifted by 128 for display purposes. 
This problem can be elegantly solved using the periodic plus smooth decomposition we proposed. Let us consider a discrete $M \times N$ image $u$ to be extended to the continuous domain $D=[0, M-1] \times[0, N-1]$, and write $(p, s)$ its periodic plus smooth decomposition. The periodic component $p$ can be extended into $\bar{p}: D \rightarrow \mathbb{R}$ with periodic sinc interpolation, that writes in Fourier domain

$$
\bar{p}(x, y)=\frac{1}{M N} \sum_{(q, r) \in R} \hat{p}(q, r) \exp \left[2 i \pi\left(\frac{q x}{M}+\frac{r y}{N}\right)\right],
$$

where $R=\mathbb{Z}^{2} \cap\left(\left[-\frac{M-1}{2}, \frac{M-1}{2}\right] \times\left[-\frac{N-1}{2}, \frac{N-1}{2}\right]\right)$. Concerning $s$, since it is a very smooth image, even a very local interpolation is convenient, so we can use a bilinear interpolation to obtain an extended image $\bar{s}: D \rightarrow \mathbb{R}$ defined by

$$
\begin{aligned}
\bar{s}(x, y)= & (x-[x])((y-[y]) s([x]+1,[y]+1)+([y]+1-y) s([x]+1,[y])) \\
& +([x]+1-x)((y-[y]) s([x],[y]+1)+([y]+1-y) s([x],[y])),
\end{aligned}
$$

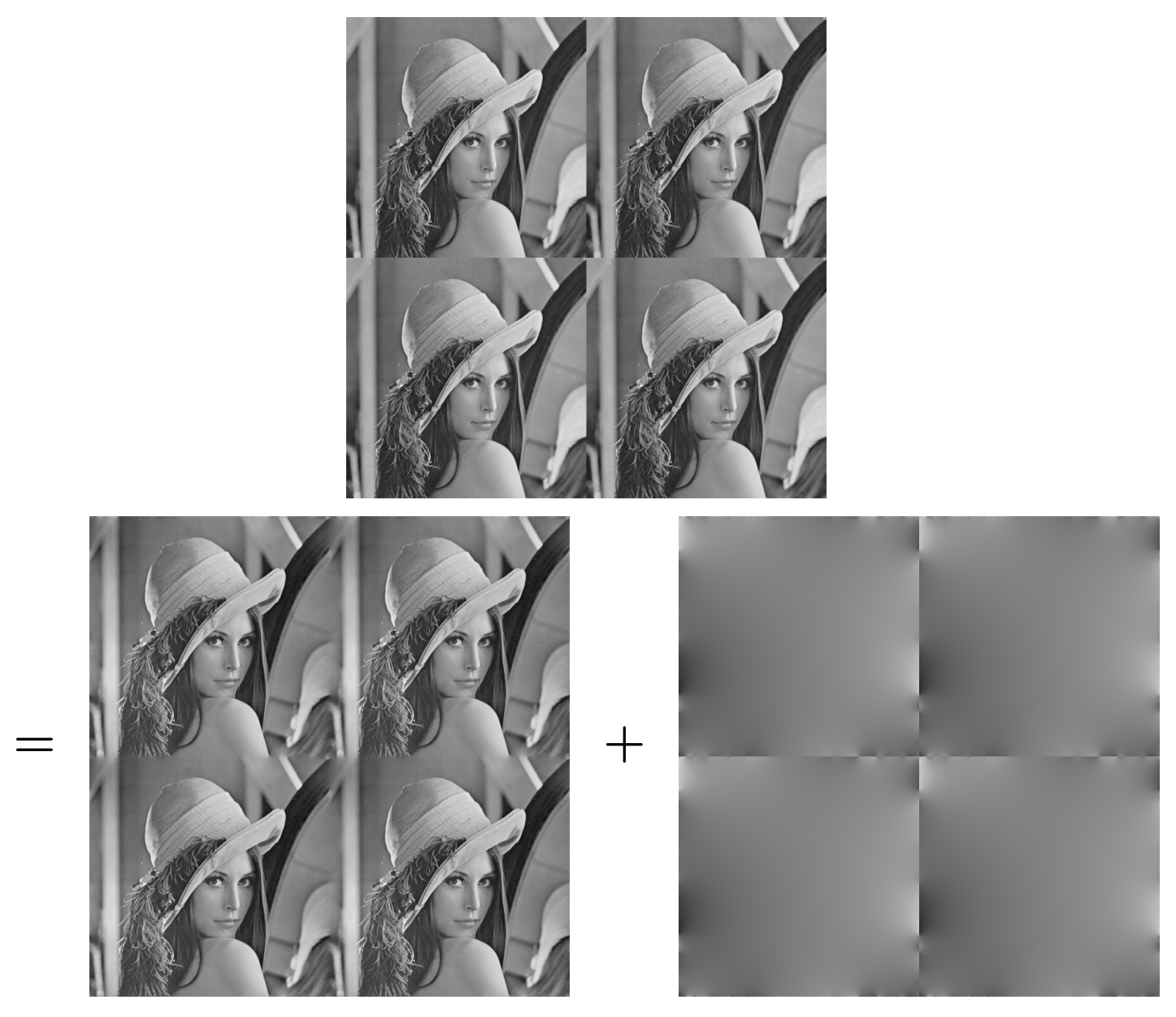

Figure 4: The effect of the decomposition $u=p+s$ on the image borders is shown by replicating the three images $u$ (top), $p$ (bottom, left) and $s$ (bottom, right) in a periodic manner. As we can see, the frame border discontinuities of $u$ are put into $s$, while the $p$ component presents smooth transitions, making the replication frame much less visible than for $u$. 

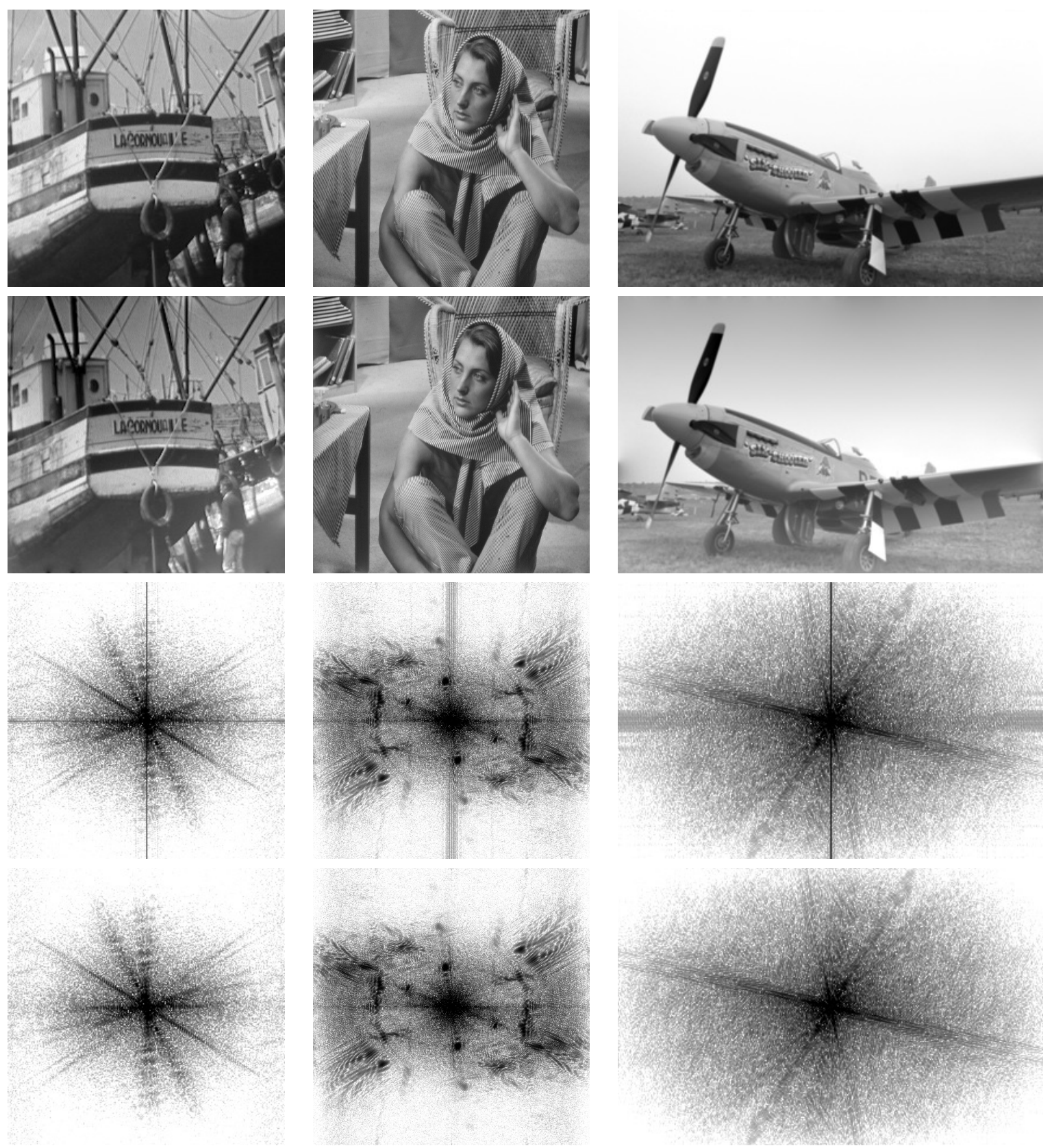

Figure 5: Effect of the per operator on three classical images (La Cornouaille, Barbara, and a resampled version of the Plane [24] image). Each column concerns a different image: the original image is displayed on the first row, its periodic component on the second row, and the corresponding Fourier spectra (modulus in log scale) are displayed on row 3 (original image) and 4 (periodic component). On each of these images, the per operator nicely removes the DFT artifacts of the Fourier spectrum, while keeping a good visual resemblance with the original image. The two main effects of the per operator in the image domain are large-scale slow contrast changes (in particular for the Plane image, that has a strong vertical contrast between its top and bottom lines), and some periodic-diffusion artifacts around the image borders (La Cornouaille, bottom line, and Plane, left column just above the skyline). 
where $[t]$ denotes the integer part of $t$. Thus, extending $u$ into

$$
\bar{u}=\bar{p}+\bar{s},
$$

we benefit from the accuracy and efficiency of periodic sinc interpolation without the ringing artifacts it generally causes. The interest of this construction for image zooming is illustrated on Figure 6.
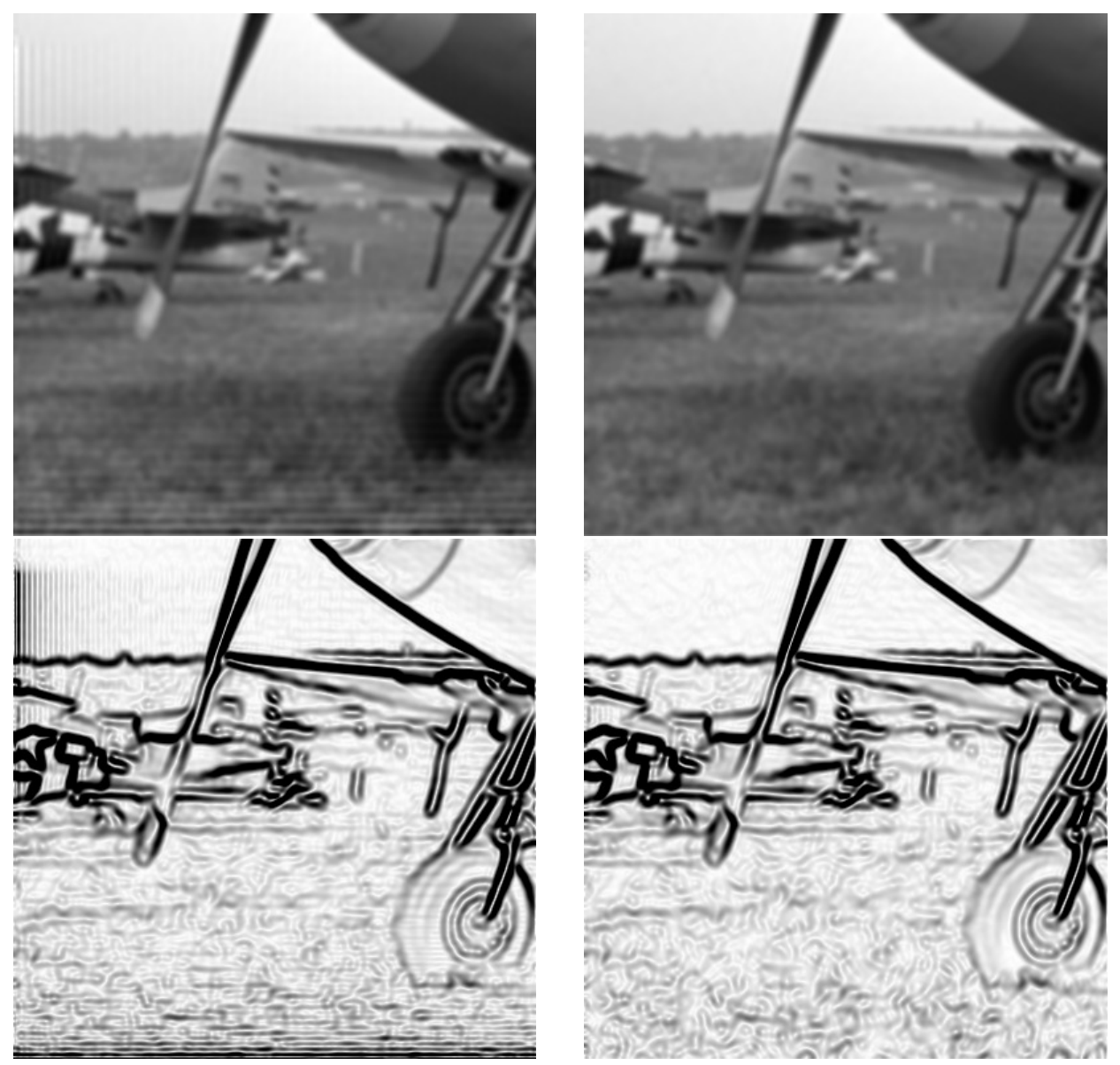

Figure 6: First row: the bottom-left corner of the Plane image (see Figure 5) has been zoomed by a factor 4 by applying a periodic cardinal sine (sinc) interpolation. The sinc interpolation was applied in two different ways: on the left column, directly to the Plane image; on the right column, to the periodic component only, the smooth component being processed with bilinear interpolation. As we can see, the ringing artifacts caused by periodic sinc interpolation (or equivalently, by the use of the DFT), are completely avoided when the periodic plus smooth decomposition is used. The effect is particularly strong on the image gradient (norm), displayed under each image (second row). This illustrates the fact that periodic plus smooth decomposition offers a way to use Shannon (sinc) interpolation without undergoing unpleasant edge effects. 


\subsection{Dequantization}

An unexpected application of Shannon interpolation is the dequantization of images. Suppose that $u$ is a discrete image that has been sampled in agreement with Shannon Theory (that is, a bandlimited image that can be well interpolated using the sinc kernel), and that, for some reason, the gray levels of $u$ have been significantly quantized. Even if the number of gray levels is not too small, quantization may cause important bias, in particular for the estimation of the local orientation of the gradient [9], or for the precise estimation of the Total Variation [4]. In [9], Desolneux et al. proposed to apply a $(1 / 2,1 / 2)$ translation (using sinc interpolation) to remove the "quantization noise" of such an image. The idea is the following: on the one hand, since the discrete image $u$ is equivalent to its continuous interpolant, and thus to any sub-pixelic translated version of $u$, a $(1 / 2,1 / 2)$ translation is harmless (it is an invertible process); on the other hand, it can be shown (see [9]) that such a translation transforms the structured quantization noise into a quasi-Gaussian noise, which is very interesting for quantization-sensitive applications. As a special case of Shannon interpolation considered above, this dequantization process can be performed efficiently using the periodic plus smooth decomposition, in such a way that edge effects are avoided without the need for a heavy symmetrization process.

On Figure 7, the La Cornouaille image (visible on Figure 5, top-left) is quantized into 20 grey levels (b), and then processed with this dequantization process in two ways. In (c), the $(1 / 2,1 / 2)$ translation is applied to the whole image, whereas in (d) only the periodic component is translated (the smooth component is kept unchanged and added afterwards). The improvement brought by the periodic plus smooth decomposition is clearly visible.

\subsection{Ringing detection}

When using Shannon (sinc) interpolation on a discrete image, ringing artifacts may appear for two reasons. If the image has not been sampled in agreement with Shannon Theory, the aliasing it suffers from is transformed into ringing artifacts by sinc interpolation, and spurious oscillations appear near the image contours (that is, highly-contrasted edges). This is a true artifact, which shows that the considered image is not compliant with Shannon Theory. However, as we saw before, even the interpolant of a Shannon-compliant image can suffer from ringing artifacts, just because of the forced periodization induced by periodic sinc interpolation. Once again, the periodic plus smooth decomposition can be used to avoid these systematic ringing artifacts and leave only potential true ringing artifacts. This idea was developed in [5] to build a ringing detector (and, by the way, a Shannon-compliance indicator), using a probabilistic acontrario model. We shall not detail this model here, but we illustrate on Figure 8 how the use of the periodic plus smooth decomposition permits, once again, to avoid artifacts (undesirable detections) on the frame border.

\subsection{Global Phase Coherence}

As we saw in Section 4.1, a natural application of the periodic plus smooth decomposition is the estimation of an artifact-free spectrum. The role of this decomposition can be essential, as in [4], where a sharpness indicator is built by measuring the Global Phase Coherence (GPC) of 


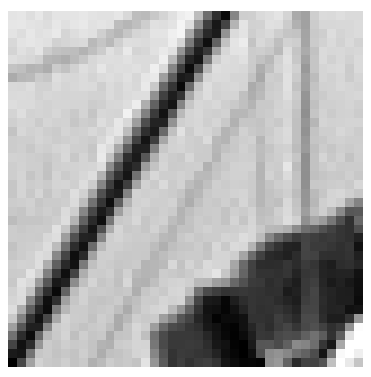

(a) original

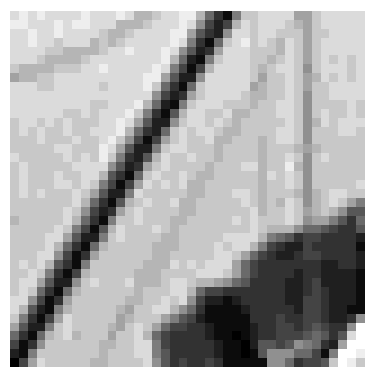

(b) quantized

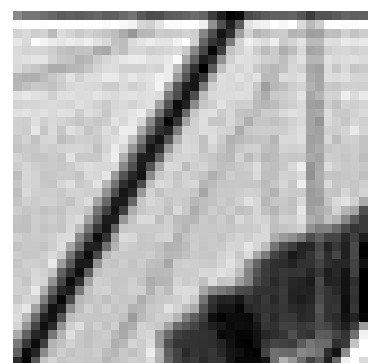

(c) dequantization $[9]$

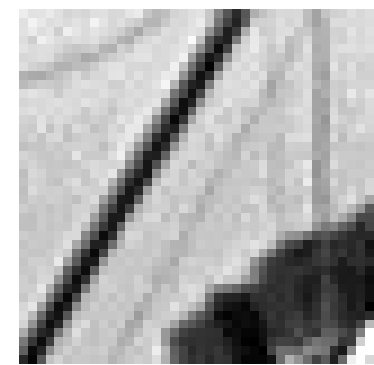

(d) dequantization [9] with $p+s$

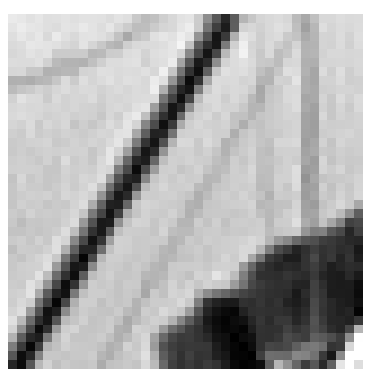

(e) reference

Figure 7: Effect of the periodic plus smooth decomposition on the dequantization process proposed in [9]. All images correspond to the $40 \times 40$ upright corner of La Cornouaille image. (a): the original $L a$ Cornouaille image; (b): same image, but quantized in 20 gray levels; (c): dequantization of (b) obtained after a $(1 / 2,1 / 2)$ translation realized directly in Fourier domain; (d): dequantization obtained by applying the $(1 / 2,1 / 2)$ translation to the periodic component only; (e): original image after a $(1 / 2,1 / 2)$ translation (reference image). As we can see, the additional periodic plus smooth decomposition improves the quality of the dequantization by removing undesirable ringing effects on the frame border, without requiring a symmetrization process.

an image. Theoretically, the GPC of an image $u$ is defined by

$$
G P C(u)=-\log _{10} \operatorname{Prob}(T V(U) \leq T V(u)),
$$

where $T V$ is the discrete Total Variation operator (see [4] for details) and $U$ is a random image obtained from $u$ by replacing the phase component of its Fourier Transform with random values (uniform and independent random variables, up to the conjugate symmetry of the Fourier Transform). However, the comparison between the regularity of $u$ and $U$ (through the TV operator) is not completely fair if the the original image $u$ is used, because of periodization artifacts that introduce a huge bias. The use of the periodic component of $u$, dequantized with a $(1 / 2,1 / 2)$ translation (as we saw in Section 4.3), permits to avoid this bias and to obtain an operational measure. In this process, the periodic component plays a crucial role for two reasons: first, the phase in Fourier domain has to be estimated properly everywhere, which is not the case with direct DFT in reason of the "cross artifact" mentioned before; second, the quantization of the original image slightly reduces its Total Variation, which introduces a bias with respect to images with random phases (that are not quantized). In this application, the periodic plus smooth decomposition hence appears as a way to explore a property of the image spectrum that could probably not be observed without handling the periodization artifacts arising from the DFT. As an illustration, we show on Figure 9 that the GPC of a blurry image is strongly overestimated when the periodic plus smooth decomposition is not used. 

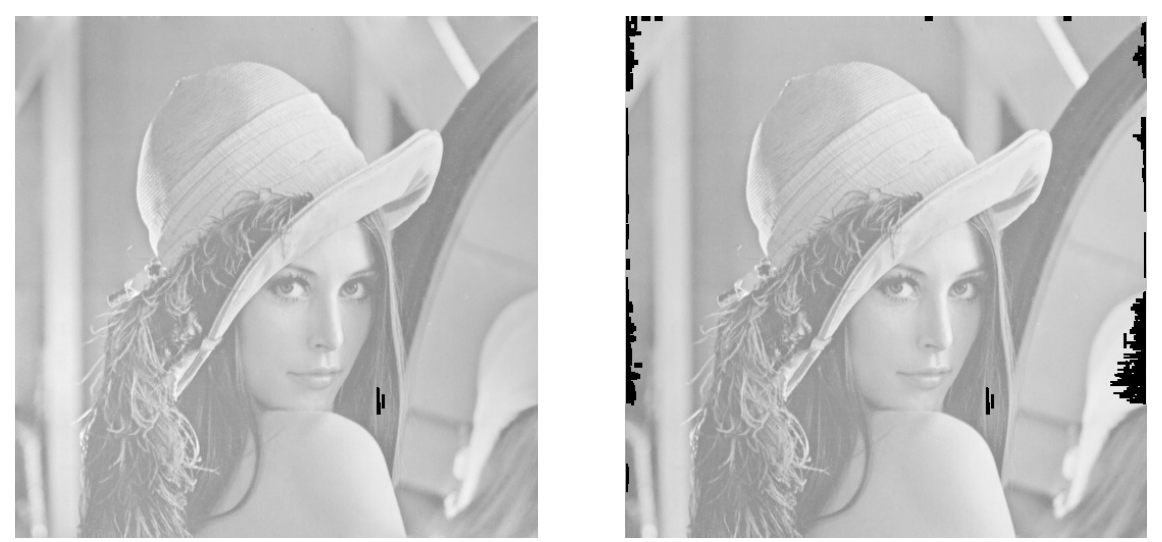

Figure 8: Effect of the periodic plus smooth decomposition on ringing detection. The classical Lena image is submitted to the Shannon-compliance test described in [5], which consists in a Shannon $(1 / 2,0)$ translation followed by an a-contrario horizontal ringing detection process (with an expected number of false alarms equal to 0.01). When the periodic component is analyzed (left), ringing detection (marked in black) only occurs in a textured zone (the hair) where horizontally oscillating pattern are expected, which supports the idea that Lena image has been sampled in accordance with Shannon Theorem. When the original image is used directly (right), a lot of spurious detections arise on the frame border.
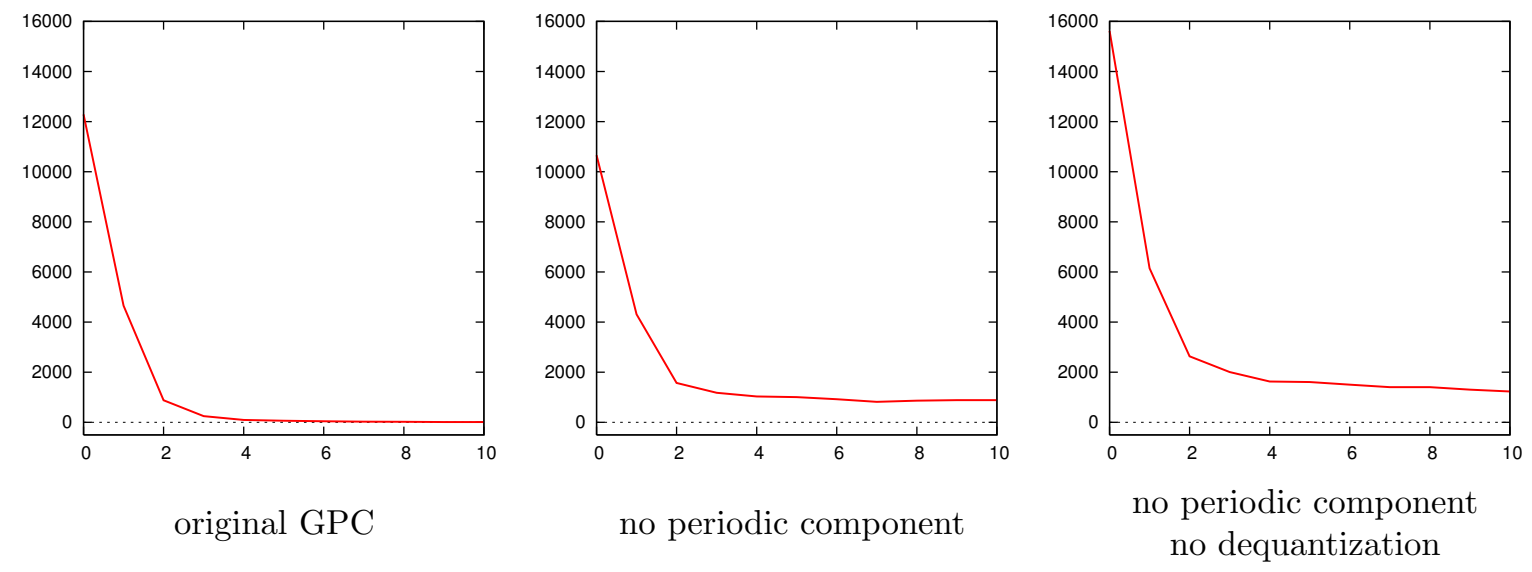

Figure 9: Importance of the periodic plus smooth decomposition in the estimation of the Global Phase Coherence [4]. The La Cornouaille image is blurred by a Gaussian kernel with increasing width $\sigma=$ $0,1,2, \ldots, 10$, and the GPC is plotted as a function of $\sigma$. Left: original GPC, as defined in [4], that uses the periodic plus smooth decomposition and the dequantization process [9]. Middle: modified GPC, that does not use the periodic plus smooth decomposition but still uses the dequantization process [9]. Right: modified GPC, that uses neither the periodic plus smooth decomposition nor the dequantization process [9]. Since GPC is a sharpness indicator, it is expected to quickly decrease to 0 as $\sigma$ grows, which is what we observe on the left graph. But as we can see, this property is lost when the periodic plus smooth decomposition is not used (middle and right graphs), in reason of the remaining intensity gap arising across the image frame border. 


\section{Conclusion}

In this paper, we proposed a simple and efficient way to deal with periodization artifacts arising from the use of the Discrete Fourier Transform on 2-D images. It consists in the decomposition of the discrete image into a periodic component, that gathers most of the image information but avoids periodization artifacts, and a smooth (harmonic) component that presents very slow variations inside the image domain. In contrast with the PHLST/PHLST5 method [22, 28] (that could be used for the same purpose, though it was primarily intended for local decomposition), the "periodic component" we propose matches more closely the original image, since it is not constrained to be null at the image frame border. We derived explicit formulas in Fourier and image domains, and showed in particular that the associated linear $u \mapsto \operatorname{per}(u)$ operator is robust to noise and that it could be iterated to yield a projector forcing an exact match of image borders. Applications of the proposed decomposition were discussed, including spectrum analysis, Shannon interpolation, and recent works related to image quality assessment.

We believe that the periodic plus smooth decomposition opens interesting perspectives concerning the systematic use of Shannon (sinc) interpolation, whose main drawback (edge effects) can be avoided by considering the periodic component. The present work could be generalized to color images (either by processing the channels independently or by using a more sophisticated distance on colors), and the extension to volumic (3-dimensional or more) images is straightforward. Variants of this work could also be easily investigated by considering other variational formulations, involving for example non- $L^{2}$ norms or a more complex neighborhood structure.

\section{Software resources}

Codes for periodic plus smooth decomposition as well as decomposition examples are freely available on the web page http://www.mi.parisdescartes.fr/ moisan.

\section{Acknowledgments}

We thank the anonymous referees for their useful comments, in particular concerning the link with Poisson's equation.

\section{References}

[1] A. Averbuch, M. Israeli, L. Vozovoi, "A fast Poisson solver of arbitrary order accuracy in rectangular regions", SIAM J. Sci. Comput. 19:3, pp. 933-952, 1998.

[2] H. H. Barrett, J. L. Denny, R. F. Wagner, K. J. Myers, "Objective assessment of image quality. II. Fisher information, Fourier crosstalk, and figures of merit for task performance", Journal of the Optical Society of America A 12:5, pp.834-852, 1995.

[3] N. Beaudoin, S. Beauchemin, "An Accurate Discrete Fourier Transform for Image Processing", Proceedings of Int. Conf. on Pattern Recognition, vol. 3, pp. 935-939, 2002. 
[4] G. Blanchet, L. Moisan, B. Rougé, "Measuring the global phase coherence of an image", Proceedings of the International Conference on Image Processing (ICIP), pp. 1176-1179, 2008.

[5] G. Blanchet, L. Moisan, B. Rougé, "Automatic detection of well sampled images via a new ringing measure", Proceedings of the International Conference on Acoustics, Speech, and Signal Processing (ICASSP), 2010.

[6] W. L. Briggs, V. E. Henson, The DFT: An Owner's Manual for the Discrete Fourier Transform, SIAM, Philadelphia, PA, 1995.

[7] J. W. Cooley, J. W. Tukey, "An algorithm for the machine calculation of complex Fourier series", Math. Comput. 19, pp. 297-301, 1965.

[8] E. De Castro, C. Morandi, "Registration of translated and rotated images using finite Fourier transforms", IEEE Transactions on Pattern Analysis and Machine Analysis 9:5, pp. 700-703, 1987.

[9] A. Desolneux, S. Ladjal, L. Moisan, J.-M. Morel, "Dequantizing image orientation", IEEE Transactions on Image Processing 11:10, pp. 1129-1140, 2002.

[10] F. Guichard, F. Malgouyres, "Total Variation based interpolation", Proc. European Signal Processing Conf. 3, pp. 1741-1744, 1998.

[11] F. J. Harris, "On the use of Windows for Harmonic Analysis with the Discrete Fourier Transform", Proceedings of the IEEE 66:1, pp. 51-83, 1978.

[12] D. He, Q. Sun, "A Practical Print-scan Resilient Watermarking Scheme", Proceedings of the International Conference on Image Processing (ICIP), pp. 257-260, 2005.

[13] D. Marr, E. Hildreth, "Theory of Edge Detection", Proceedings of the Royal Society London 207, pp. 187-217, 1980.

[14] L. A. Hutchison, W. A. Barrett, "Fourier-Mellin registration of line-delineated tabular document images", International Journal on Document Analysis and Recognition 8:2-3, pp. 87-110, 2006.

[15] C. Kuglin, D. Hines, "The Phase Correlation Image Alignment Method", Proc. Int. Conf. Cybernetics and Society, pp. 163-165, 1975.

[16] J. Larrey-Ruiz, R. Verdu-Monedero, J. Morales-Sanchez, "A Fourier Domain Framework for Variational Image Registration", Journal of Mathematical Imaging and Vision 32:1, 2008.

[17] S. Leprince, S. Barbot, F. Ayoub, J.-P. Avouac, "Automatic and precise orthorectification, coregistration, and subpixel correlation of satellite images, application to ground deformation measurements", IEEE Transactions on Geoscience and Remote Sensing 45:6, pp. 1529-1558, 2007.

[18] C.-Y. Lin, Min Wu, J. A. Bloom, I. J. Cox, M. L. Miller, Yui Man Lui, "Rotation, Scale, and Translation Resilient Watermarking for Images", IEEE Trans. Image Processing 10:5, pp. 767-782, 2001. 
[19] A. W. Lohmann, D. Mendelovic, G. Shabtay, "Significance of phase and amplitude in the Fourier domain", J. Opt. Soc. Am. A 14, pp. 2901-2904, 1997.

[20] N. B. Nill, B. H. Bouzas, "Objective image quality measure derived from digital image power spectra", Opt. Eng. 31:4 , pp. 813-825, 1992.

[21] B. S. Reddy, B. N. Chatterji, "An FFT-based technique for translation, rotation, and scaleinvariant image registration", IEEE Transactions on Image Processing 5:8, pp. 1266-1271, 1996.

[22] N. Saito, J.-F. Remy, "The Polyharmonic Local Sine Transform: A New Tool for Local Image Analysis and Synthesis without Edge Effect", Applied and Computational Harmonic Analysis 20:1, pp. 41-73, 2006.

[23] C. E. Shannon, "Communication in the presence of noise", Proceedings of the Institute of Radio Engineers 37:1, pp. 10-21, 1949.

[24] H. R. Sheikh, Z. Wang, L. Cormack, A. C. Bovik, LIVE Image Quality Assessment Database Release 2, http://live.ece.utexas.edu/research/quality, 2005.

[25] Bugao Xu, "Identifying Fabric Structures with Fast Fourier Transform Techniques", Textile Research Journal 66:8, pp. 496-506, 1996.

[26] Min Xu, P. K. Varshney, Ruixin Niu, "A Subspace Method for Fourier Based Image Registration", Asilomar Conference on Signals, Systems and Computers, pp. 425-429, 2006.

[27] K. Yamatani, N. Saito, "Improvement of DCT-Based Compression Algorithms Using Poisson Equation", IEEE Transactions on Image Processing 15:12, pp. 3672-3689, 2006.

[28] J. Zhao, N. Saito, Y. Wang, "PHLST5: A Practical and Improved Version of Polyharmonic Local Sine Transform", J. of Mathematical Imaging and Vision 30:1, pp. 23-41, 2008.

[29] Xiao-Qun Zhang, J. Froment, "Total variation based Fourier reconstruction and regularization for computer tomography", Nuclear Science Symposium Conference Record 4, pp. 2332-2336, 2005. 